\title{
Leveraging Zebrafish to Study Retinal Degenerations
}

\author{
Juan M. Angueyra ${ }^{1 *}$ and Katie S. Kindt ${ }^{2 *}$ \\ ${ }^{1}$ Retinal Neurophysiology Section, National Eye Institute, National Institutes of Health, Bethesda, MD, United States, \\ ${ }^{2}$ Section on Sensory Cell Development and Function, National Institute on Deafness and Other Communication Disorders, \\ National Institutes of Health, Bethesda, MD, United States
}

\section{OPEN ACCESS}

Edited by:

Gokhan Dalgin

The University of Chicago,

United States

Reviewed by:

Glenn Prazere Lobo,

Medical University of South Carolina,

United States

Deborah Stenkamp,

University of Idaho, United States

*Correspondence:

Juan M. Angueyra

angueyra@nih.gov

Katie S. Kindt

katie.kindt@nih.gov

Specialty section:

This article was submitted to

Molecular Medicine,

a section of the journal

Frontiers in Cell and Developmental

Biology

Received: 28 May 2018

Accepted: 20 August 2018

Published: 19 September 2018

Citation:

Angueyra JM and Kindt KS (2018)

Leveraging Zebrafish to Study

Retinal Degenerations.

Front. Cell Dev. Biol. 6:110.

doi: 10.3389/fcell.2018.00110
Retinal degenerations are a heterogeneous group of diseases characterized by death of photoreceptors and progressive loss of vision. Retinal degenerations are a major cause of blindness in developed countries (Bourne et al., 2017; De Bode, 2017) and currently have no cure. In this review, we will briefly review the latest advances in therapies for retinal degenerations, highlighting the current barriers to study and develop therapies that promote photoreceptor regeneration in mammals. In light of these barriers, we present zebrafish as a powerful model to study photoreceptor regeneration and their integration into retinal circuits after regeneration. We outline why zebrafish is well suited for these analyses and summarize the powerful tools available in zebrafish that could be used to further uncover the mechanisms underlying photoreceptor regeneration and rewiring. In particular, we highlight that it is critical to understand how rewiring occurs after regeneration and how it differs from development. Insights derived from photoreceptor regeneration and rewiring in zebrafish may provide leverage to develop therapeutic targets to treat retinal degenerations.

Keywords: zebrafish, retinal degeneration, regeneration, photoreceptor cells, Müller glia, developmental biology, rewiring, retinal circuitry and visual pathways

\section{RETINA AND PHOTORECEPTORS}

Similar to many organs, the eye is structurally well-conserved between zebrafish and mammals. For example, the eyes of zebrafish have the same gross structure as human and other mammalian eyes, and contain a cornea, lens, vitreous, retina, pigment epithelium, choroid and sclera (Figure 1A). Furthermore, the development of the eye during embryogenesis is also conserved, and complimentary work in zebrafish, mice and other species has helped to delineate the key developmental events in eye morphogenesis across vertebrates (Bibliowicz et al., 2011; Stenkamp, 2015).

Within the eye, the retina is of particular interest because it is the site of sensory detection and damage to the retina results in vision loss. The vertebrate retina is a highly structured neuronal tissue that lines the back of the eye. It is responsible for both the detection and processing of visual information, before it is relayed to higher-order visual centers. To achieve this, the retina is equipped with a variety of neurons that are arranged into three nuclear layers and project into two synaptic layers (Figures 1B,C). Within these layers, retinal neurons assemble into multiple, distinct circuits that encode different aspects of the visual information (Gollisch and Meister, 2010). The encoding of visual information starts when light is detected by the rod and cone photoreceptors. The highly sensitive rods are mainly used during dim-lighting conditions, while the more adaptable but less sensitive cones function from dawn until dusk. The retina of the nocturnal rodents commonly 

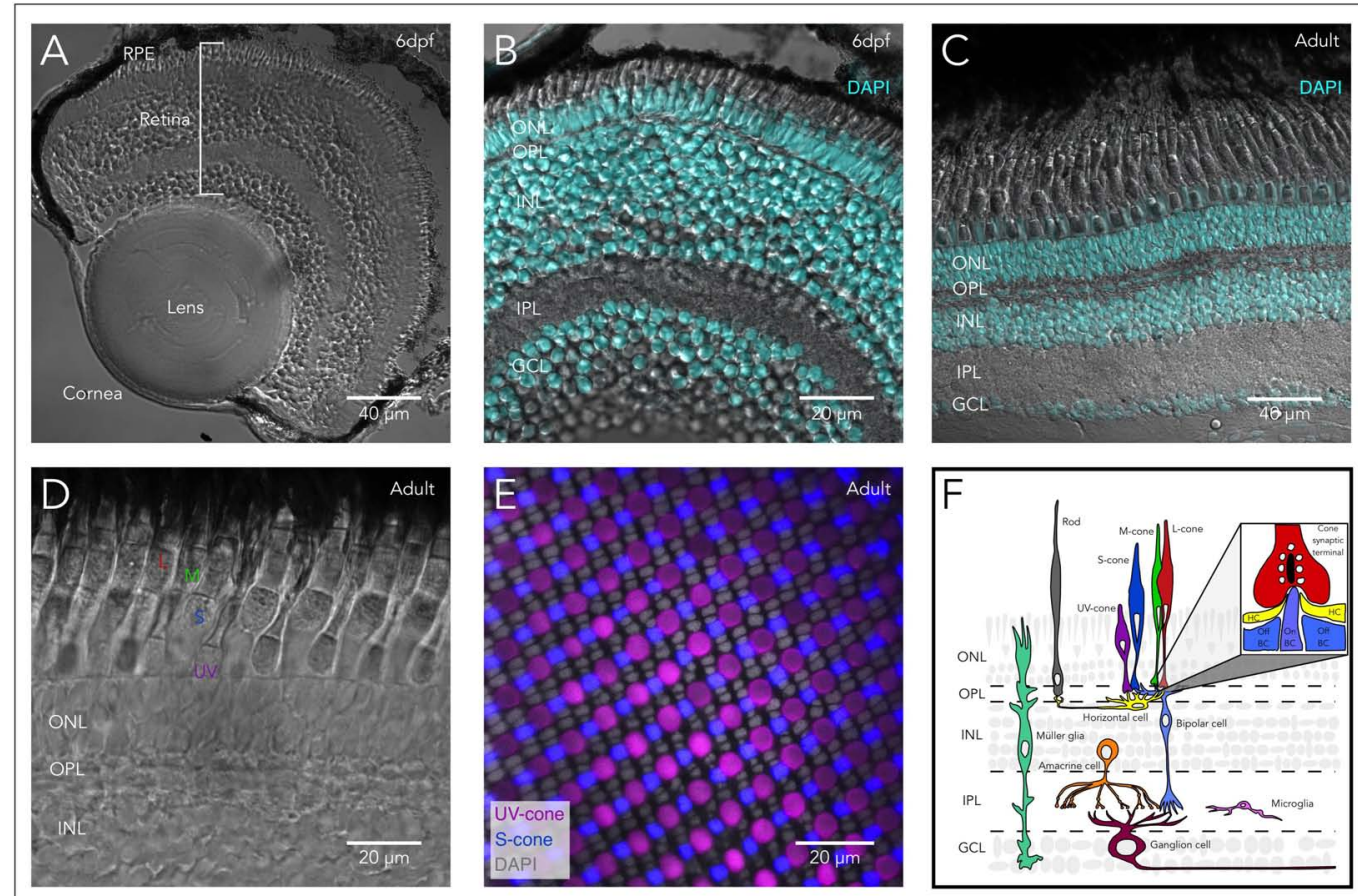

FIGURE 1 | Structure of the zebrafish eye and retina. (A) Anatomy of the zebrafish eye: DIC image of a cryosection from a 6 days post-fertilization (6 dpf) larval eye highlighting the main structures of the vertebrate eye including cornea, lens, retina, and retinal pigment epithelium (RPE). (B) The larval retina is organized into highly structured layers: Overlay of a DIC image and the fluorescent nuclear marker DAPI of a cryosection from a 6 dpf larva showing the different retinal layers, including the outer nuclear layer (ONL) which contains the cell bodies of photoreceptors (rods and cones). Photoreceptors make synapses in the outer plexiform layer (OPL) with bipolar and horizontal cells. The inner nuclear layer (INL) contains the cell bodies of horizontal, bipolar and amacrine cells, while the ganglion cell layer (GCL) contains the cell bodies of retinal ganglion cells (RGC). Bipolar cells provide excitatory synaptic input to RGC in the inner plexiform layer (IPL), while amacrine cells modulate this input both pre- and post-synaptically. (C) The adult retina retains the same layered structure: Overlay of a DIC image and DAPI of a cryosection from an adult zebrafish. (D) The zebrafish retina contains 4 subtypes of cones: DIC image of a cryosection from an adult zebrafish showing the short-single or ultraviolet-wavelength sensitive cones (UV-cones), the long-single or short-wavelength sensitive cones (S-cones), and the double cones which correspond to the middle- and long-wavelength sensitive cones (M- and L-cones). (E) Mosaic arrangement of zebrafish cone photoreceptors: Confocal image of a whole-mounted retina of a double-reporter transgenic lines to identify UV-cones [Tg(sws1:GFP) ${ }^{k j 9}$, magenta] and S-cones [tg(sws2:mCherry) ${ }^{\text {uaz011 }}$, blue] overlayed with DAPI (gray), allowing the identification of the nuclei of $\mathrm{M}$ - and L-cones between the rows of UV- and S-cones. (F) Diagram of the vertebrate retina and the retinal cells. Inset highlights the synapse between cones and horizontal and bipolar cells, where the cone synaptic terminal contains synaptic vesicles (white) attached to the synaptic ribbon (black). In close apposition to the ribbon, the dendrites on On-bipolar cells (On-BCs) invaginate into the synaptic terminal and are flanked by two horizontal cell (HC) processes. Off-bipolar cells make more basal contacts in close proximity but not apposed to the synaptic ribbon.

used in research like mice and rats is rod dominated $(97 \%$ rods and 3\% cones) (Carter-Dawson Louvenia and Lavail Matthew, 1979), as is the peripheral human retina. In contrast, zebrafish have a cone-dominated retina ( $\sim 40 \%$ rods and $\sim 60 \%$ cones) (Fadool, 2003), similar to the central human retina, which provides high-acuity vision, and is essential for most day-today visual tasks. Therefore, the zebrafish retina is uniquely positioned to understand the molecular mechanisms relevant to development and regeneration of the photoreceptors that are most relevant for human vision.

The zebrafish retina contains four different cone photoreceptor subtypes (UV-, S-, M-, and L-cones). Each subtype is defined by specific opsin expression that confers a particular wavelength-sensitivity, and morphology (short or long, and single or paired with another cone type) (Figures 1D,E). UV-cones express $s w s 1$, an opsin with peak sensitivity $\left(\lambda_{\max }\right)$ in the ultraviolet range $\left(\lambda_{\max }=354 \mathrm{~nm}\right)$, and are short-single cones morphologically. S-cones express $s w s 2$, with peak sensitivity at short wavelengths $\left(\lambda_{\max }=416 \mathrm{~nm}\right)$, and are long-single cones morphologically. M-cones express opsins of the Rh2 class, which have undergone tandem quadruplications (Rh2-1 to Rh2-4), with peak sensitivities at mid wavelengths $\left(\lambda_{\max }=467 \mathrm{~nm}, 476 \mathrm{~nm}\right.$, $488 \mathrm{~nm}$, and $505 \mathrm{~nm}$ respectively). L-cones express one of two tandemly duplicated opsin genes from the lws class, with peak sensitivities at longer wavelengths $\left(\lambda_{\max }=548 \mathrm{~nm}\right.$ and $\left.558 \mathrm{~nm}\right)$. $\mathrm{M}$ - and L-cones are morphologically arranged as a double cone, where the L-cone is the long (or principal) member of the pair and the M-cone is the short (or accessory) member (Raymond et al., 1996; Vihtelic et al., 1999; Chinen et al., 2003).

In both rod and cone photoreceptors, light triggers the activation of opsins followed by the rest of the phototransduction cascade. In zebrafish and in mammals, this cascade ultimately leads to changes in photoreceptor membrane potential, and to modulation of neurotransmitter release in the synaptic terminal. Visual information is then directly transmitted from photoreceptors to several subtypes of horizontal (inhibitory 
interneurons that locally modulate photoreceptor synaptic output) and bipolar cells (glutamatergic neurons that transmit light signals into the next processing layer). Information is further processed in the next synaptic layer, where bipolar cells (BC) provide excitation to ganglion cells (glutamatergic spiking neurons), while amacrine cells (local interneurons) provide modulation pre- and/or post-synaptically. The axons of the retinal ganglion cells (or RGCs) form the optic nerve, and relay the pre-processed visual information to central targets in the brain. Additionally, the retina contains microglia (resident immune cells located primarily in the synaptic layers) and two types of true glial cells: Müller cells (a type of radial glia) and astrocytes (associated with axons of RGCs) (Figure 1F). The photoreceptors also closely associate with the retinal pigment epithelium (RPE), which provides structural, trophic and metabolic support and is directly involved in the recycling of opsins.

\section{RETINAL DEGENERATIONS}

Within the retina, rods and cones are particularly vulnerable to metabolic, genetic or environmental insults; phototransduction and synaptic signaling are demanding processes that require high metabolic rates. Retinal degenerations (RD) are disorders characterized by photoreceptor death and can affect both rods and cones, or each photoreceptor type individually. RD have multiple causes that can be broadly divided based on whether the cause is primary or secondary. In primary $\mathrm{RD}$, photoreceptors are directly affected, for example due to mutations that affect phototransduction proteins. In secondary $\mathrm{RD}$, other cells types, for example the RPE or a single type of photoreceptor degenerates; this can lead to secondary degeneration of other photoreceptors. The main forms of RD in humans are Age-related Macular Degeneration (AMD), Retinitis Pigmentosa (RP), and Leber Congenital Amaurosis (LCA). AMD is a multi-factorial disease that affects the RPE. Advanced RPE dysfunction leads to secondary rod and cone loss. In industrialized countries, AMD is the leading cause of blindness (De Bode, 2017). AMD is associated with age, smoking, nutritional deficiencies, inflammation and mutations or polymorphisms in more than 30 genes, with many more genes still to uncover (Jager et al., 2008; Warwick and Lotery, 2018). RP encompasses a set of complex hereditary diseases that can be caused by a plethora of mutations mapped to more than 70 human genes (Daiger et al., 2013; Farrar et al., 2017) ${ }^{1}$. Most forms of RP in humans are characterized by an initial death of rods, with subsequent secondary cone death. RP presents clinically first with night-blindness, followed by decreases in peripheral visual acuity (tunnel vision) that eventually progresses toward the central areas of the retina. LCA encompasses a group of early-onset and progressive rod and cone dystrophies. Again, 25 genes have already been identified as causes of LCA, and most are expressed either in photoreceptors or in the RPE (Kumaran et al., 2017).

\footnotetext{
${ }^{1}$ http://www.sph.uth.tmc.edu/retnet
}

Early forward genetic screens in zebrafish that evaluated defects in the histology of the photoreceptor layer identified many genes that cause RD (Brockerhoff and Fadool, 2011). Most human homologs of these genes have also been shown to cause $\mathrm{RD}$ in humans. Subsequent screens in zebrafish assayed for defects in visual function rather than morphological defects. These screens exploited reliable visual behavioral assays in larvae, including the optokinetic response (OKR) (Brockerhoff et al., 1995 ) or the escape response to moving dark objects ( $\mathrm{Li}$ and Dowling, 1997) (see below). Together these screens helped, not only to establish additional genetic models for photoreceptor degenerations, but also to identify additional and in some cases novel genes involved in RD. For example, one zebrafish mutant that lacked OKR was linked to a mutation in pde6c, a novel cone-specific phototransduction gene. Mutations in pde6c cause cone degeneration in zebrafish with secondary rod degeneration (Stearns et al., 2007). The discovery of this mutant was beneficial in two ways. First pde6c mutants were used to confirm the existence of rod-specific progenitors in the zebrafish retina (Morris et al., 2008). Second this mutant was used to identify $p d e 6 c$ as the causative gene in cone-photoreceptor loss of function 1 (cpfl1), a specific type of cone degeneration in mouse and humans (Chang et al., 2009). This is an excellent example of how zebrafish can be used to identify novel genes and pathways involved in RD. More recently, zebrafish models of RD are being leveraged in pharmacological screens, to test or find novel treatments for human RD (Moosajee et al., 2008; Ganzen et al., 2017). Overall these zebrafish genetic screens highlight the conservation of molecules underlying RD between zebrafish and mammals. Given this level of conservation, it is likely similar molecules may be required to regenerate and rewire the zebrafish and mammalian retina after $\mathrm{RD}$.

\section{THERAPIES FOR RD}

In humans, photoreceptor loss in $\mathrm{RD}$ is permanent (regardless of their diverse causes and speed of progression) and therefore remain largely untreatable and lead to progressive loss of vision and ultimately blindness. In early stages of $\mathrm{RD}$ in humans, current treatments include neuroprotective agents (Trifunovic et al., 2012) and antibody therapies in cases where the underlying mutation is well characterized (Lazic and Gabric, 2007). Unfortunately, these treatments only slow down the progression of disease and have variable outcomes (Pardue and Allen, 2018). In addition to these treatments, gene therapy has been used to improve vision in patients with LCA caused by mutations in RPE65, an RPE-specific protein involved in the recycling of retinoids (Cideciyan, 2010; Jacobson et al., 2012), but the improvement may not be long-lasting (Jacobson et al., 2015).

In late stages of RD in humans, when there is widespread photoreceptor loss, two distinct approaches for treatment exist. The first seeks to bypass the need for photoreceptors. This can be achieved by either making the surviving retinal bipolar or ganglion cells photosensitive using optogenetics (Busskamp et al., 2010; Yue et al., 2016) or synthetic photo-switchable compounds (Polosukhina et al., 2012; Tochitsky et al., 2017). Additionally, 
retinal prostheses capable of stimulating RGCs directly have been developed, attempting to encode visual information directly into these output neurons (da Cruz et al., 2016; Lewis et al., 2016). Of note, the use of retinal prostheses for blindness was approved by the European Union in 2011, and by the FDA in 2013. Use of these prostheses has led to some successful reacquisition of very basic visual functions but only for limited periods of time (Mills et al., 2017). The second and more promising approaches aims to replace the lost photoreceptors by transplantation or by stimulating regeneration. These later approaches have received special attention because they have the capability of renewing the native function of the retina, and could provide a real cure for RD. Due to this potential for complete functional recovery, and because of recent and important developments in the field, transplantation and regeneration will be a focus of this review.

\section{BARRIERS IN PHOTORECEPTOR TRANSPLANTATION AS A THERAPY FOR RD}

Just a decade ago, the prospect of producing photoreceptors from stem cells seemed like an overly daunting task (Adler, 2008). Nevertheless, in the last few years, several laboratories have successfully developed protocols to produce eyecup-like structures from induced-pluripotent stem cells (iPSCs) in the span of weeks. Some of these eyecups are able to acquire a layered structure reminiscent of the retina, with photoreceptorlike cells that contain outer segments, express phototransduction proteins (Wahlin et al., 2017), and have some capacity for light responsiveness (Zhong et al., 2014), and vesicular release (Wahlin et al., 2017). The successful development of these eyecups opens the possibility of harvesting cells from an individual to generate iPSCs, and re-differentiate them into photoreceptors that could be then transplanted back into patients with RD. Based on these prospects, recent work in the retinal field has focused on using mice to explore photoreceptor transplantation as a therapy for RD.

Initial transplantation studies in mice attempted to introduce rod photoreceptors, with the best rates of integration (never surpassing a few percent) achieved by transplanting immature rod precursors (MacLaren et al., 2016). Follow-up studies presented equally promising examples of integration, and in some cases demonstrated functional recovery of vision (SantosFerreira et al., 2015; Smiley et al., 2016). However, it has recently been discovered that many of these results are due to the exchange of cytoplasmic material (including RNA and/or proteins) between donor cells and the host retina, and not integration of transplanted photoreceptors (Pearson et al., 2016; Santos-Ferreira et al., 2016; Singh et al., 2016; Ortin-Martinez et al., 2017). In light of this recent discovery, it will be important to carefully interpret how functional recovery of vision occurred after transplantation/cytoplasmic exchange in degenerated or degenerating retinas (Homma et al., 2013; Singh et al., 2013; Santos-Ferreira et al., 2015). Even if cytoplasmic exchange results in recovery photoreceptor function, it occurs at very low rates (a few percent of host positive cells, for transplantations of tens to hundreds of thousands of donor cells). Such low rates casts doubt on the prospect of leveraging this process as a viable therapeutic strategy, especially in advanced cases of degeneration.

Even with viable evidence for successful photoreceptor transplantation (Waldron et al., 2018), there are additional concerns for this type of therapy. For example, subretinal injection of a mass of cells, the most common transplantation method, causes inflammation and scarring, and inhibits the migration of transplanted cells (Barber et al., 2013). Additionally, it is also unclear if transplanted photoreceptors are capable of rewiring properly into the host retina. This problem is further compounded by our incomplete understanding of how photoreceptors normally wire during development. To date, only a handful of genes are known to be involved in synapse formation between photoreceptors and their postsynaptic targets (Simmons et al., 2017; Zhang et al., 2017; Miller et al., 2018; Sarria et al., 2018; Ueno et al., 2018). Despite this work, we still have little insight on the molecules that drive the initial recognition between these cells, or on the processes that promote, inhibit or refine synapse formation. Unveiling genes involved in photoreceptor synapse formation during normal development in zebrafish could provide direct therapeutic targets to promote rewiring of transplanted photoreceptors.

\section{USING ZEBRAFISH TO EXPLORE RETINAL REGENERATION AS A THERAPY FOR RD}

Cumulatively, work on transplantation therapies has highlighted that alternative therapies, such as photoreceptor regeneration, could be a promising alternative. Unfortunately, in mammals there is no regeneration in the retina after damage or $\mathrm{RD}$. In contrast, the zebrafish retina has the innate capacity for regeneration. This capacity may be due to the continued growth of the zebrafish retina into adulthood, as well as the ability of the zebrafish to maintain a population of multipotent stem cells within the retina.

Larval zebrafish form a functional visual system by 4 days post fertilization ( $4 \mathrm{dpf}$ ), and are able to perform complex visual guided behaviors (like prey capture, see below) by $5 \mathrm{dpf}$ (Patterson et al., 2013). This rapid onset of sensory function is critical to survival of the animal. As larvae progress into adulthood, zebrafish continue to grow in size. This growth requires organs like the eye and retina to grow as well. In the retina this growth occurs in the ciliary marginal zone (CMZ). The CMZ maintains a niche of pluripotent cells at the edge of the retina that continually adds neurons in peripheral concentric rings (Centanin et al., 2011). In addition to this continual growth, zebrafish can also regenerate their retinas after injury. In fact, robust retinal regeneration and rewiring have been demonstrated in genetic models of $\mathrm{RD}$ and in other models that incur retinal injury. Overall, given that zebrafish is a genetically tractable model with active retinal regeneration, it is poised to uncover the molecular processes that control retinal regeneration and rewiring. 
In teleost fish, retinal regeneration after injury has a rich history. It was first reported in goldfish (Lombardo, 1968) and later in cichlids (Johns and Fernald, 1981) and trout (Julian et al., 1998). In zebrafish retinal regeneration is robust after resection (Cameron, 2000), mechanical damage (Fausett and Goldman, 2006), light damage (Bernardos et al., 2007; Thomas et al., 2012), thermal damage (Raymond et al., 2006), pharmacological damage (Fimbel et al., 2007; Sherpa et al., 2008; Nagashima et al., 2013; Tappeiner et al., 2013; Sherpa et al., 2014) and selective ablation of particular cell-types (Montgomery et al., 2010; D’Orazi et al., 2016; Hagerman et al., 2016; Yoshimatsu et al., 2016; White et al., 2017). In teleosts, regeneration can occur from cells generated in the CMZ (Raymond et al., 2006), a dedicated population of progenitors that are committed to a rod fate (Bernardos et al., 2007; Morris et al., 2008), and the Müller glia (Fausett and Goldman, 2006; Bernardos et al., 2007; Fimbel et al., 2007).

Due to its location at the edge of the retina, the CMZ is only involved in regeneration if the injury involves the peripheral retina. During regeneration, the $\mathrm{CMZ}$ is capable of giving rise to all retinal neurons except rod photoreceptors (Stenkamp et al., 2001; Raymond et al., 2006). Instead, rods originate from rodspecific progenitors. These progenitors were first identified in goldfish and cichlids (Johns and Fernald, 1981) and were later found in other teleost fish including trout and zebrafish (Julian et al., 1998). Rod-specific progenitors are thought to be important for maintaining the density of rods as the eye grows, and lineage tracing revealed that these rod progenitors derive from Müller cells that slowly and continuously divide in the normal retina (Otteson et al., 2001; Raymond et al., 2006; Bernardos et al., 2007; Nelson et al., 2008). During regeneration, there is an expansion in the number of photoreceptor progenitors, but these mainly derive from actively dividing Müller glia. In fact, in zebrafish the Müller glia are the primary source of regenerated neurons after injury. During regeneration they can act as multipotent stem cells, dividing and differentiating into any retinal cell type (Ramachandran et al., 2010b). In contrast to zebrafish, in humans and other mammals Müller glia do not remain multipotent and therefore cannot readily replace lost neurons in the retina. Because Müller glia are the primary source of regenerated retinal neurons and can regenerate all retinal neurons, considerable work has been dedicated to understanding the differences between the Müller glia of zebrafish and mammals.

A series of studies that investigated the response of zebrafish Müller glia to retinal injury, have unveiled the key transcription factors in a gene regulatory network that controls retinal repair. Shortly after injury, cytokines and growth factors activate the beta-catenin and stat3 pathways (Kassen et al., 2007; Wan et al., 2014). These pathways upregulate the expression of ascl1 (Fausett et al., 2008), a key transcription factor that (through lin-28) leads to the suppression of let7 microRNA (Ramachandran et al., 2010a). In the uninjured retina, let7 normally represses the expression of many regeneration-induced genes (including ascl1 and lin-28), closing the loop of a system poised to control Müller glia response to injury (Wan and Goldman, 2016) (Figure 2A).

In contrast, mammalian Müller glia does not readily divide (Wan et al., 2008) and responds to retinal injury with an inflammatory response known as reactive gliosis, characterized by an increase in size and overproduction of intermediate filaments, and leading to distortion of the architecture of the retina without repair (Dyer and Cepko, 2000; Bringmann et al., 2009; Thomas et al., 2016) (Figure 2C). Significant efforts have been devoted to understanding the differences between these species, in the hope of stimulating regeneration in mammals. This work has shown that ascl1 is not upregulated in mice after retinal injury (Karl et al., 2008), but ascl1 overexpression in mammalian Müller cells in vitro is sufficient to induce production of neurons (Pollak et al., 2013). Moreover, induction of expression of ascl1 in Müller cells in vivo, followed by retinal injury, induces division and production of all classes of retinal neurons, but only in young mice (Ueki et al., 2015). Recently, a successful report of regeneration in adult mice has shown that new bipolar- and amacrine-like cells derived from Müller glia can rewire into the retina. For this work, in addition to overexpression of ascl1, inhibition of histone deacetylation was also required (Jorstad et al., 2017). Unfortunately, no other retinal cell types are produced with this protocol (Figure 2D). Further insight into retinal regeneration has been gained from studying a related telost, medaka, which shows a restricted capacity for regeneration. In medaka fish, after injury, Müller glia do not readily proliferate, and new retinal progenitors commit almost exclusively to a photoreceptor fate. Comparisons in the Müller glia response to retinal injury in both medaka fish and zebrafish concluded that sustained expression of the transcription factor sox2 in adult Müller cells is key for maintaining multipotency (Lust and Wittbrodt, 2018) (Figure 2B). While it is clear that important strides have been taken to attempt retinal regeneration in mammals, before regeneration can be used as a viable therapy, we need a deeper understanding on the mechanisms that maintain cells with a regenerative potential in zebrafish throughout adulthood.

\section{INSIGHTS FROM ZEBRAFISH ON REWIRING AFTER REGENERATION}

Even after successful transplantation or regeneration of photoreceptors, the biggest hurdle in these $\mathrm{RD}$ therapies is ensuring that the new photoreceptors rewire into the appropriate retinal circuits so that they are able to restore normal visual function. Once again, zebrafish has offered a unique opportunity to study rewiring after injury and regeneration, in both larvae and adults. Cumulatively, this work has demonstrated that the extent and time course of regeneration and rewiring is determined by lesion-specific differences, in particular the extent of injury and the number of cells that need to be replaced.

Adult teleosts are able to regenerate their retinas even after extensive retinal damage. Early seminal studies in adult goldfish, and later studies in adult zebrafish, showed robust retinal regeneration and rewiring after surgical retinal extirpation (Hitchcock et al., 1992; Cameron, 2000) or pharmacologically induced death of all retinal neurons (Raymond et al., 1988; Sherpa et al., 2008). Under these lesion paradigms, all retinal cell types were regenerated. Importantly, with regards to rewiring, the retinal lamination was reestablished (Raymond et al., 1988; 

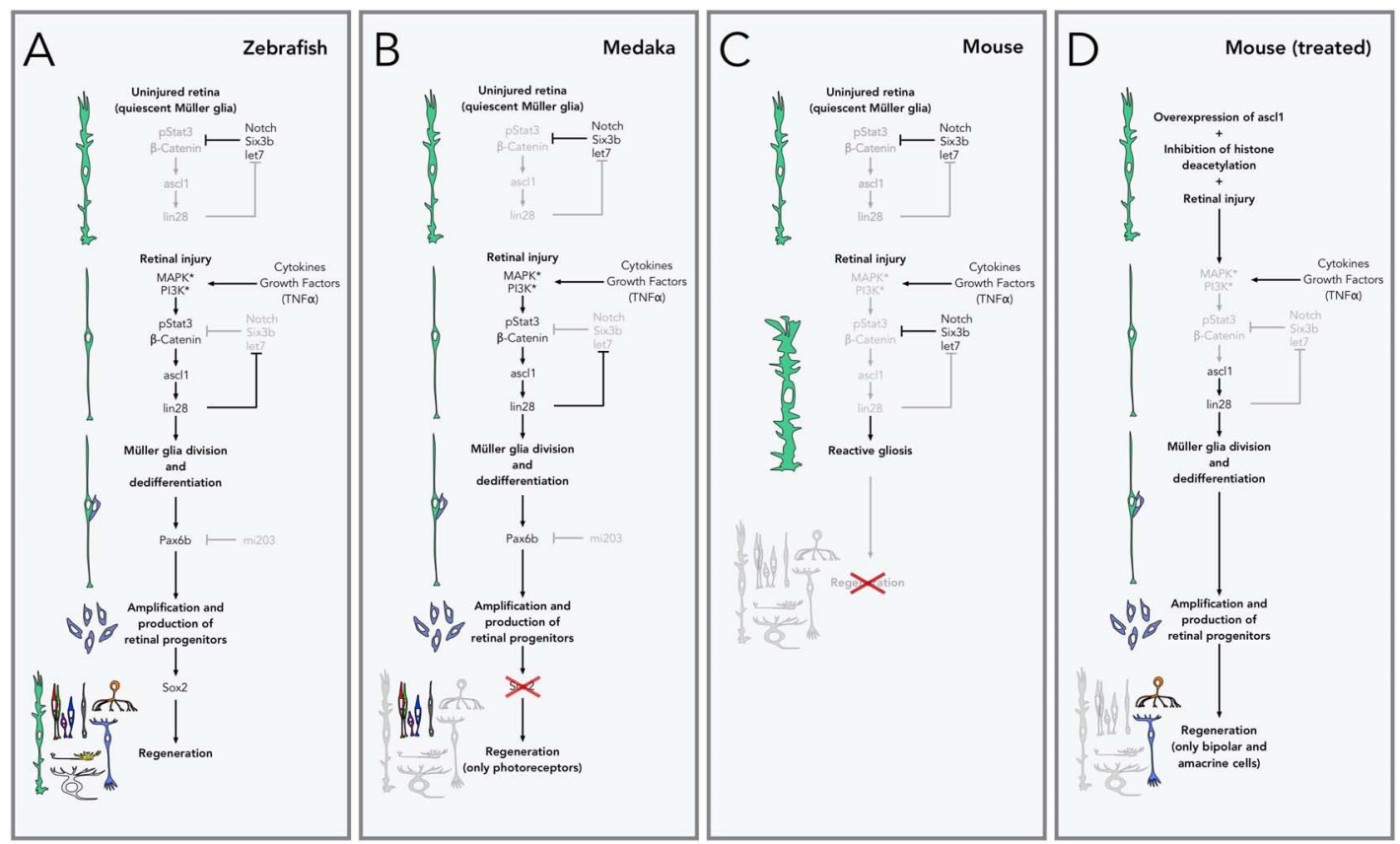

FIGURE 2 | Pathways to retinal regeneration. (A) Zebrafish are able to completely regenerate their retina via Müller glia. In the uninjured retina, Müller glia is kept quiescent by inhibiting the expression of the genes that control regeneration through Notch signaling and repression by the transcription factor Six $3 \mathrm{~b}$ and the microRNA let7, amongst others. During retinal injury, cell death and inflammation lead to the release of cytokines and growth factors (especially TNF $\alpha$ ), which activate receptors and kinases in the Müller glia, leading to activation of $\beta$-catenin and phosphorylation of the transcription factor Stat3, which in turns leads to the production of the transcription factor asc/1 and lin28 microRNA, and the activation of the genes that control Müller glia division and dedifferentiation. lin28 also inhibits the production of let7, releasing the inhibition of this pathway. Expression of the transcription factor Pax6b (normally inhibited by the microRNA mi203), allows the amplification and production of retinal progenitors which are then able to redifferentiate into any retinal neurons or Müller glial cells. (B) In medaka fish, lack of production of Sox2 after the production of retinal progenitors restricts their fate to photoreceptors and does not allow the production of other cell types, including new Müller glia. (C) In mice and other mammals, retinal injury does not lead to expression of asc/1 and the rest of regeneration-related genes. Instead Müller glia activate the production of intermediate filaments and increase their size (reactive gliosis) and do not lead to the production of new retinal cells or injury repair. (D) Retinal regeneration can be stimulated in mice by artificially overexpressing asc/1 and inhibiting epigenetic changes (histone deacetylation in particular). With this treatment, Müller glial cells are able to divide and produce retinal progenitors, but their fate is restricted to bipolar and amacrine cells.

Sherpa et al., 2008), and synaptic connections were reformed between retinal neurons (Hitchcock and Cirenza, 1994) and between RGCs and the brain (Stuermer et al., 1985). Parallel work demonstrated that after extensive retinal damage in adult goldfish and zebrafish, visual function is also recovered (Mensinger and Powers, 1999, 2007; Lindsey and Powers, 2007; Sherpa et al., 2008). Nevertheless, because the Müller glia are the main source of regenerated retinal neurons, complete ocular excision prevents regeneration (Mensinger and Powers, 2007).

In adult teleosts, although there is robust regeneration after extensive retinal injury, the time required for regeneration and functional recovery depends on the extent of injury. For example, the differences in regeneration and rewiring were examined after surgical extirpation of $\sim 25,50$, and $75 \%$ of the adult retina. This work found that after surgical extirpation of $25 \%$ of the retina, 17 weeks (120 days) are required for regeneration and reestablishment of lamination and 25 weeks (180 days) for functional recovery. Additional extirpation lengthened the time and extent of recovery for both lamination and functional recovery (Mensinger and Powers, 2007). Similar results were observed in adults after pharmacological damage and death of all retinal neurons (induced by high doses of intraocular ouabain).
In this study functional recovery started 5 weeks after the injury with further improvement by weeks $7-10$, albeit with decreased sensitivity (Mensinger and Powers, 1999; Lindsey and Powers, 2007).

In adult zebrafish, the extent and time course of retinal regeneration and rewiring is also dependent on the injury. Extensive pharmacological damage of all retinal layers with ouabain leads to regeneration, and the newly formed cells are capable to reorganize into the three distinct nuclear layers by week 3 after injury. After 14 weeks, the regenerated retinas are well laminated (clear nuclear and plexiform layers), the optic nerve has regrown, and there is functional recovery (Sherpa et al., 2008). Interestingly, with pharmacological damage (using lower doses of ouabain) that spares photoreceptors and Müller glia but still induces a loss of cells in the INL and GCL, regeneration is faster, with significant recovery of function after only 8 weeks (Sherpa et al., 2014; McGinn et al., 2018). In this lesion paradigm, rewiring of a specific (but heterogeneous) subset of regenerated $\mathrm{BCs}$ was closely examined. The regenerated BCs had largely normal morphology, and, as a population, were able to reproduce the diversity of connectivity patterns observed in the surviving photoreceptors, with only a few errors in lamination or abnormal 
dendritic or axonal arborization, again emphasizing the robust regeneration of zebrafish (McGinn et al., 2018). Nevertheless, in the context of extensive damage, regeneration in adult teleosts is far from perfect. Several structural defects are common including areas with defects in the formation or absence of plexiform layers, disorganization of nuclear layers, presence of cells in the incorrect layer (e.g., RGCs in INL), failure to reestablish the photoreceptor mosaic, formation of photoreceptor rosettes, overproduction of neurons, and the generation of cell types that were not initially damaged (Raymond et al., 1988; Hitchcock et al., 1992; Cameron, 2000; Vihtelic Thomas and Hyde David, 2000; Stenkamp et al., 2001; Stenkamp and Cameron, 2002; Sherpa et al., 2008; Powell et al., 2016).

More recently, the genetic tractability of zebrafish has enabled researchers to damage specific retinal cell types and study their rewiring after regeneration. This work was accomplished by using the recently developed nitroreductase-metronidazole (NTR-MTZ) system. For this method, transgenic zebrafish are created expressing the NTR gene $(n f s b)$ under the control of a cell-specific promoter. When these transgenic zebrafish are treated with the compound MTZ, the NTR converts MTZ into a cytotoxic compound. Because this compound does not diffuse to neighboring cells, the resulting ablation is restricted to the NTR-expressing cells. Importantly, this process is reversible, and removal of MTZ solution makes it possible to examine regeneration and rewiring. To date, the NTR-MTZ system has been used to selectively ablate rods, and specific subtypes of cones, bipolar cells and glial cells (Zhao et al., 2009; Ariga et al., 2010; Montgomery et al., 2010; Fraser et al., 2013; D’Orazi et al., 2016; Hagerman et al., 2016; White et al., 2017). Importantly, several of these studies demonstrated that other cells in the retina that did not express NTR were not ablated after MTZ application. In addition, these treatments did not affect the surrounding retinal architecture (Zhao et al., 2009). This highlights the specificity and power of the NTR-MTZ system.

After genetic ablation and removal of MTZ, in each instance the targeted cells regenerated after several days, although the exact time-course varied depending on the ablated cell type and the age of zebrafish treated. For example, after using the NTR-MTZ to completely ablate rods in adult zebrafish, newly generated rods were identified within a week after removal of MTZ, and repopulation of rods attained pre-injury levels within 4 weeks (Montgomery et al., 2010) - a very similar time course required for the regeneration of cones in adults (Raymond et al., 2006; Bernardos et al., 2007). In larvae, the regeneration of cells occurs at a faster timescale. For example, after ablation of rods using the NTR-MTZ system in $5 \mathrm{dpf}$ larvae, newly formed rods attained control levels in just 6 days (White et al., 2017). Similarly, cones ablated between 4 and $6 \mathrm{dpf}$ regenerate in $7-10$ days (Fraser et al., 2013; Yoshimatsu et al., 2016) and BCs ablated at 7 dpf regenerate in 13 days (D'Orazi et al., 2016).

In the majority of these studies, regeneration was confirmed morphologically. In a subset of studies, after regeneration, the analysis was extended to include behavior or rewiring. For example, in one study, either the UV- or S-cones were ablated (in $7 \mathrm{dpf}$ larvae) and the optomotor response (OMR) (see below) was assayed after ablation and following UV- or S-cone regeneration respectively (Hagerman et al., 2016). The OMR was reduced immediately after ablation of either UV- or S-cones. Surprisingly, while the OMR recovery took 4 days for the UV-cone ablation, the OMR recovered in just 1 day following S-cone ablation, before new S-cones were produced. These differences in behavioral recovery suggest that there may be a capacity for plasticity amongst the remaining cells, used to compensate for the ablated cells during the recovery phase. It is possible that this shortterm plasticity relies on activity from other cone subtypes and/or on synaptic remodeling. Evidence for such synaptic remodeling has been reported in a parallel study (Yoshimatsu et al., 2016). In this study, a subtype of horizontal cell (H3) that normally connects preferentially to UV- and S-cones, was able to reconnect to UV-cones after UV-cone specific ablation and regeneration. Nevertheless, if UV-cone regeneration was delayed, the $\mathrm{H} 3$ made additional contacts with S-cones and even $\mathrm{M}$ - and L-cones, suggesting functional compensation at the level of rewiring. Another recent study examined rewiring after selective loss of a subpopulation of BC using the NTR-MTZ system in $5 \mathrm{dpf}$ larvae (D'Orazi et al., 2016). Thirteen days after the ablation of these $\mathrm{BC}$, the majority of regenerated $\mathrm{BC}$ were morphologically normal but the rewiring did not fully recapitulate development, with a relative loss of selectivity for specific cone subtypes. Additionally, BC axons contained significantly more synapses.

As a whole, work in this field proves that retinal regeneration in zebrafish is a robust process, but also suggests that some of the developmental cues required to refine synapse number or proportion of photoreceptor subtypes innervated may not be present during regeneration. In the future, it will be important to further understand what cues are present during development that enable photoreceptors to wire into different retinal circuits. It will be especially important to understand how specific photoreceptor subtypes recognize the different bipolar- and horizontal-cell subtypes, and the factors required for the formation of these synapses. It will also be important to recognize and examine the developmental and environmental differences between larval and adult zebrafish retinas. This knowledge will provide a comprehensive understanding of the differences between development and regeneration, between wiring, rewiring and remodeling, and likely uncover manipulations that could be used to modify or refine rewiring in the context of treatments for RD.

\section{THE ZEBRAFISH TOOLKIT FOR THE STUDY OF RETINAL DEVELOPMENT AND REGENERATION}

There are numerous factors that have established zebrafish as a valuable model organism for the study of human disease including rapid development, large clutch sizes, ease of maintenance, genetic conservation, accessibility to genetic manipulations, and optical transparency of embryos. In addition to these advantages, we have described several examples of how zebrafish has been a useful model to investigate retinal development and regeneration. To aid in these studies, multiple tools have been developed that have direct application for the 
study of retina in zebrafish. We have summarized some of these tools here as a convenient reference.

\section{Imaging Retinal Cells and Their Connectivity}

Currently there are established transgenic lines that make it straightforward to visualize each cell subtype within the retina, as well as the lamination and precise wiring of these cells (Table 1). For example, taking advantage of the specificity of opsin expression in the different photoreceptor subtypes, promoters from each opsin have been utilized to create transgenic lines that label rods (rods: rho) (Fadool, 2003) and each of the four cone subtypes (UV-cones: opn1sw1; S-Cones: opn1sw2; M-Cones: opn1mw2; L-Cones: opn1lw1) (Takechi et al., 2003, 2008; Tsujimura et al., 2007, 2010) (Figures 3A,B). Additionally, multiple lines exist to label horizontal and BC. Within the retina, horizontal cells can be specifically labeled exploiting the promoter for connexin 55.5 (Weber et al., 2014; Klaassen et al., 2016), or using an enhancer-trap line that labels a combination of horizontal and amacrine cells (Torvund et al., 2017). BC represent a more diverse cell class. On-BC can be labeled using the promoter for grm $6 b$, a metabotropic glutamate receptor that is key for the detection of glutamate release by photoreceptors (Glasauer et al., 2016) (Figure 3C), and different subtypes of $\mathrm{BC}$ have been labeled with enhancer-trap lines (D’Orazi et al., 2016), or using promoters for transcription factors (Vitorino et al., 2009) or for other bipolar-specific proteins (Schroeter et al., 2006). The promoter for gfap (glial fibrillar acidic protein) can be used to label Müller glia (Raymond et al., 2006; Bernardos et al., 2007). Additionally, lines that use the promoter for mpeg1 label all macrophages (Ellett et al., 2011), allow visualization of the retinal microglia and macrophages in both the normal and regenerating retina (Mitchell et al., 2018).

Most of these lines allow high-resolution imaging of not only the lamination but also the synaptic terminals of these distinct cells types (Noel and Allison, 2018) (Figure 3D). For example, in some of these transgenic lines, the connections of photoreceptors have been used to accurately track wiring during development (Yoshimatsu et al., 2016) or rewiring after regeneration (D'Orazi et al., 2016). They can also be adapted for live imaging, allowing connections to be dynamically tracked during circuit formation or during the integration of regenerated neurons into functional retinal circuits (Ariga et al., 2010; Duval et al., 2013). Using the same promoters, several transgenic lines have been developed to express the NTR gene $(n f s b)$ and ablate specific subsets of retinal cells (see above, Table 1). This approach has enabled the study of regeneration and rewiring after selective ablation (Zhao et al., 2009; D'Orazi et al., 2016; Yoshimatsu et al., 2016; McGinn et al., 2018; Noel and Allison, 2018). Lastly, it is straightforward to perform sparse labeling to capture the fine detail of individual retinal neurons and their contacts during development or regeneration, by using transient expression in larvae (with the same promoters leveraged for transgenic lines) (Klaassen et al., 2016; Yoshimatsu et al., 2016). Alternatively, inorganic fluorescent dyes like DiI can be used in larvae or in adults (Figures 3E,F) (Connaughton et al., 2004; Li et al., 2009; Li et al., 2012).

While transgenic lines represent powerful tools to visualize cells and processes within the retina in situ, antibodies against specific markers have been identified that can also be exploited to label subsets of retinal cells (Table 2). In addition to antibodies, cones can also easily be labeled using fluorescently-tagged peanut agglutinin (or PNA), a lectin protein that binds to the cone sheath (Hageman and Johnson, 1986; Shi et al., 2017). In combination with the transgenic lines outlined above, these labels can be used to visualize multiple types of cells simultaneously within the retina (Figure 3C). In addition to simply marking specific retinal cell types, antibodies against synaptic markers are of particular usefulness to characterize retinal wiring. In photoreceptors most presynaptic markers label components of the photoreceptor ribbon synapse, like Ribeye or Syntaxin, or adjoining structures like the voltage-gated calcium channels or the synaptic vesicles (Huang et al., 2012; Lv et al., 2012; Daniele et al., 2016). Postsynaptically, photoreceptor synapse markers include: components of the postsynaptic density itself (e.g., MAGUK) and glutamate receptors (grm6 for ON-bipolars, gria4 for OFF-bipolars, gria2 for horizontal cells) (Yazulla and Studholme, 2001) (Figure 3D). These synaptic markers are extremely important for understanding the correct development of synapses or the correct rewiring of photoreceptors after regeneration. For example, alterations in the synapses between cones and Off-BC caused by mutations in pappaa, a protein recently identified in a behavioral screen, were identified by labeling the photoreceptor synaptic vesicles, but could not be seen by labeling specific cell-types, as retinal lamination was not altered (Miller et al., 2018).

\section{Functional and Behavioral Methods to Assess the Zebrafish Retina}

One of the most exploited assays for visual function in humans and many animal models are electroretinograms (ERG). ERG measure bulk electrical signals produced by the whole retina in response to light stimulation and has been adapted to both zebrafish larvae (Nelson and Singla, 2009; Chrispell et al., 2015) and adults (Hughes et al., 1998). Through analysis of the different ERG waves the overall activity of photoreceptors and $\mathrm{BC}$ can be evaluated. Using well-designed stimuli or pharmacological agents, other properties like the kinetics of photoreceptor adaptation can also be measured (Korenbrot et al., 2013). Additionally, ERG signals have a spectral signature based on the signals generated by specific subsets of photoreceptor and their downstream partners. These signatures can be utilized to isolate the contributions of each element in different conditions (Nelson and Singla, 2009). ERG measurements have been used in the adult to demonstrate that the retina can recover function after damage and subsequent regeneration (McGinn et al., 2018). In the future ERG could be used both in larvae and adults after NTRMTZ ablation of specific cells, to assess the functional recovery of retinal processing during regeneration.

While the ERG can provide a powerful readout of retinal activity, functional imaging using genetically encoded calcium 
TABLE 1 | Toolkit for the study of retinal development and regeneration: transgenic lines.

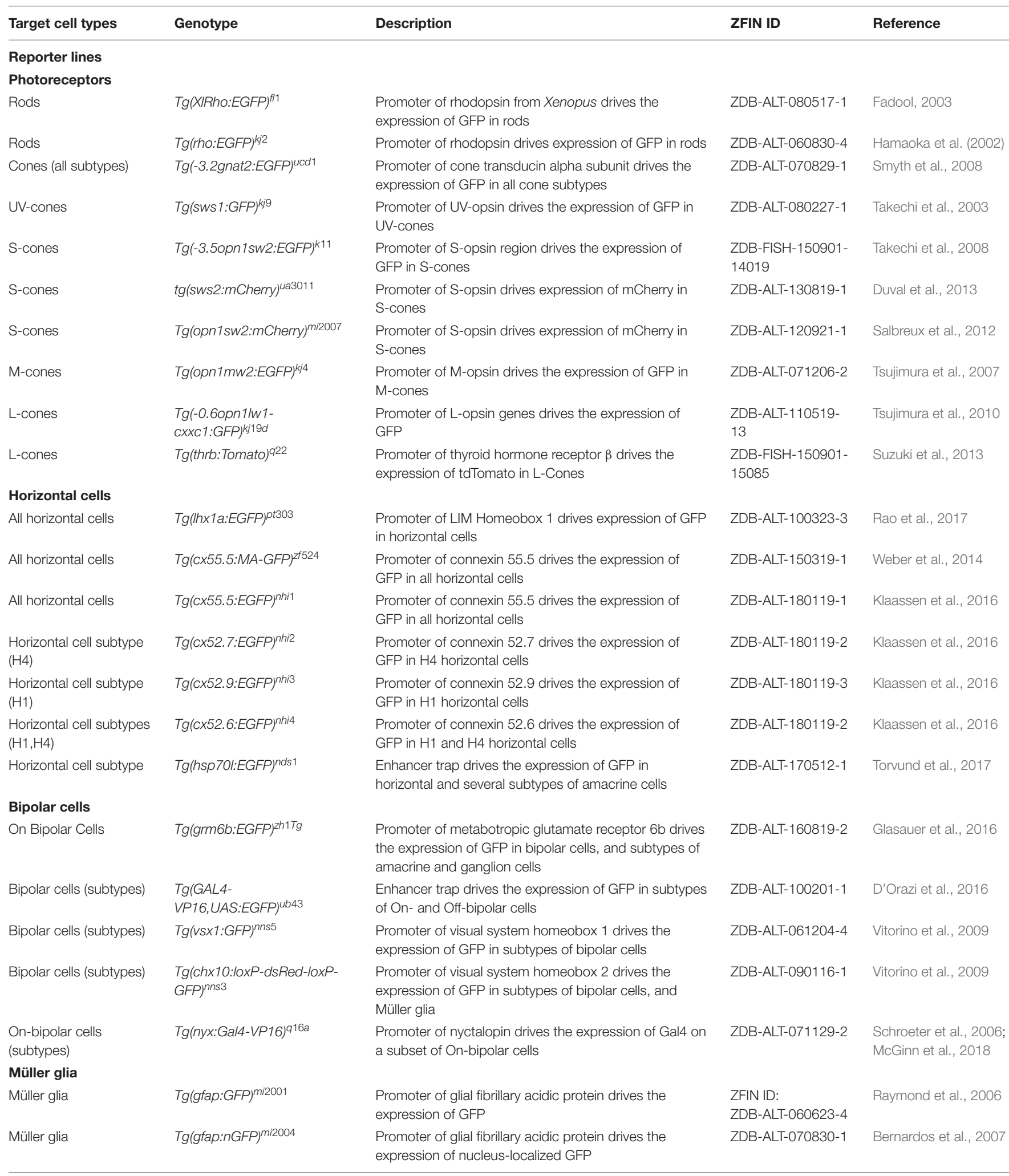


TABLE 1 | Continued

\begin{tabular}{|c|c|c|c|c|}
\hline Target cell types & Genotype & Description & ZFIN ID & Reference \\
\hline \multicolumn{5}{|l|}{ Immune cells } \\
\hline $\begin{array}{l}\text { Microglia and } \\
\text { macrophages }\end{array}$ & $\operatorname{Tg}(m p e g 1: E G F P)^{g / 22}$ & $\begin{array}{l}\text { Promoter of macrophage-expressed gene } 1 \text { drives the } \\
\text { expression of GFP }\end{array}$ & ZDB-ALT-120117-1 & $\begin{array}{l}\text { Ellett et al., 2011; } \\
\text { Mitchell et al., } 2018\end{array}$ \\
\hline $\begin{array}{l}\text { Microglia and } \\
\text { macrophages }\end{array}$ & $\operatorname{Tg}(m p e g 1: m \text { Cherry })^{g / 23}$ & $\begin{array}{l}\text { Promoter of macrophage-expressed gene } 1 \text { drives the } \\
\text { expression of mCherry }\end{array}$ & ZDB-ALT-120117-2 & $\begin{array}{l}\text { Ellett et al., 2011; } \\
\text { Mitchell et al., } 2018\end{array}$ \\
\hline \multicolumn{5}{|c|}{ Lines for selective ablation (NTR-MTZ) } \\
\hline Rods & $\operatorname{Tg}(z o p: n f s b-E G F P)^{n t 19}$ & $\begin{array}{l}\text { Promoter of rhodopsin drives the expression of } \\
\text { nitroreductase-GFP fusion in rods }\end{array}$ & ZDB-ALT-100323-4 & $\begin{array}{l}\text { Montgomery et al., } \\
2010\end{array}$ \\
\hline UV-cones & $\operatorname{Tg}(\text { opn1sw1:KALTA4) })^{\text {ua3139 }}$ & $\begin{array}{l}\text { Promoter of UV-opsin drives the expression of KalTA4, } \\
\text { which binds to a separate transgene and drives } \\
\text { expression of a nitroreductase and mCherry fusion } \\
\text { protein in UV-cones }\end{array}$ & $\begin{array}{l}\text { ZDB-ALT-160901- } \\
14\end{array}$ & Hagerman et al., 2016 \\
\hline UV-cones & $\begin{array}{l}\text { Tg(opn1sw1:NTR- } \\
\text { mCherry) }\end{array}$ & $\begin{array}{l}\text { Promoter of UV-opsin drives the expression of a } \\
\text { nitroreductase and mCherry fusion protein in UV-cones }\end{array}$ & ZDB-ALT-160425-1 & Yoshimatsu et al., 2016 \\
\hline S-cones & $\begin{array}{l}\text { Tg(opn1sw2:NTR- } \\
\text { mCherry)q30 }\end{array}$ & $\begin{array}{l}\text { Promoter of S-opsin drives expression of } \\
\text { nitroreduactase-mCherry fusion in S-cones }\end{array}$ & ZDB-ALT-160425-3 & $\begin{array}{l}\text { D'Orazi et al., 2016; } \\
\text { Yoshimatsu et al., } 2016\end{array}$ \\
\hline Bipolar cells & $\begin{array}{l}\text { Tg(UAS-E1b:NfsB- } \\
\text { mCherry) })^{c 264}\end{array}$ & $\begin{array}{l}\text { Enhancer trap drives the expression of a nitroreductase } \\
\text { and mCherry fusion protein in a subset of bipolar cells }\end{array}$ & ZDB-ALT-070316-1 & $\begin{array}{l}\text { Zhao et al., 2009; } \\
\text { D'Orazi et al., } 2016\end{array}$ \\
\hline \multicolumn{5}{|c|}{ Lines for functional imaging } \\
\hline $\begin{array}{l}\text { Photoreceptors and } \\
\text { Bipolar Cells }\end{array}$ & $\begin{array}{l}\text { Tg(-1.8ctbp2:Rno.Syp- } \\
\text { GCAMP) }\end{array}$ & $\begin{array}{l}\text { Promoter of ribeye drives synaptically localized } \\
\text { GCaMP2 in photoreceptors and bipolar cells }\end{array}$ & ZDB-ALT-120320-5 & Dreosti et al., 2009 \\
\hline $\begin{array}{l}\text { Photoreceptors and } \\
\text { Bipolar Cells }\end{array}$ & $\begin{array}{l}\text { Tg(-1.8ctbp2a:Rno.Syp- } \\
\text { GCaMP6) })^{\text {uss } 1}\end{array}$ & $\begin{array}{l}\text { Promoter of ribeye drives synaptically localized } \\
\text { GCaMP6 in photoreceptors and bipolar cells }\end{array}$ & $\begin{array}{l}\text { ZDB-ALT-161010- } \\
18\end{array}$ & Johnston et al., 2014 \\
\hline $\begin{array}{l}\text { Photoreceptors and } \\
\text { Bipolar Cells }\end{array}$ & $\operatorname{Tg}(-1.8 c t b p 2: S Y P H Y)^{I m b 2}$ & $\begin{array}{l}\text { Promoter of ribeye drives synaptic phluorin in } \\
\text { photoreceptors and bipolar cells }\end{array}$ & ZDB-ALT-120320-4 & Odermatt et al., 2012 \\
\hline Müller glia & $\begin{array}{l}\text { Tg(gfap:Eco.GltL- } \\
\text { cpEGFP)сu3313 }\end{array}$ & $\begin{array}{l}\text { Promoter of glial fibrillary acidic protein drives the } \\
\text { expression of iGluSnfR }\end{array}$ & $\begin{array}{l}\text { ZDB-ALT-170404- } \\
12\end{array}$ & MacDonald et al., 2017 \\
\hline
\end{tabular}

sensors has also been developed to measure response in visual centers, especially in the tectum (Förster et al., 2017). Currently these approaches remain challenging in the retina due to the RPE which creates an optical barrier, making it difficult to image directly through the eye. It is possible to use these indicators to image through the lens in adults (Duval et al., 2013). Also, in larvae some transparency can be achieved using PTU to inhibit melanophore production, but this treatment may alter visual function (Antinucci and Hindges, 2016). Better imaging has been achieved with mutant lines that genetically remove the different classes of pigmented cells (White et al., 2008; Antinucci and Hindges, 2016). In the future, additional functional imaging using calcium sensory in photoreceptors along with newly developed neurotransmitter sensors, like iGluSnfR (Marvin et al., 2013), will be an important in vivo approach to assess pre- and post-synaptic function with in developing and regenerating retinal circuits. (Zhang et al., 2016; MacDonald et al., 2017).

In addition to electrically or optically recording the activity of cells within the retina, there are many well characterized visual behaviors that can be used to evaluate retinal function. The optokinetic response is an extremely robust behavioral assay, where a visual stimulus of moving stripes is tracked by eye movements. This behavior is already present and reliable by 5 $\mathrm{dpf}$ and requires minimal equipment to setup (Brockerhoff et al., 1995; Neuhauss et al., 1999; Neuhauss, 2003). In the related optomotor response, tracking of moving stripes is followed by swimming in the same direction as the stimulus (Neuhauss et al., 1999; Neuhauss, 2003). This assay has been used to characterize the overall recovery of vision after photoreceptor ablation (Hagerman et al., 2016). At around 5 dpf, larvae also start hunting for small prey using visual cues, another visual behavior that can be quantified (Borla et al., 2002; Gahtan et al., 2005; McElligott and O'Malley, 2005). Larvae also innately exhibit phototaxis and photoavoidance (Brockerhoff et al., 1995; Orger and Baier, 2005; Burgess et al., 2010), and an escape response in response to sudden decreases in illumination (Burgess and Granato, 2007).

Adult zebrafish also exhibit an escape response to threatening objects, characterized by rapid turning and swimming away from the threat. The escape response can be elicited by placing fish in a clear tank with a central pole that serves for hiding, and an external rotating drum with a single black stripe to act as a threatening stimulus. Use of this assay allowed to measure behavioral rod and cone thresholds and the time course of photoreceptor adaptation and as part of the screening in a forward-genetic screen for visual mutations ( $\mathrm{Li}$ and Dowling, 1997; Li and Dowling, 2000). Zebrafish and other teleosts determine their body position using a combination of their sense of balance and the source of illumination, which in their natural environment tends to come from above. Thus, they tend to tilt their bodies such that their backs are turned against the source of illumination (dorsal light response or DLR). Tilt can be induced by uneven-illumination between the two eyes (e.g., side illumination) (Silver, 1974; Neuhauss, 2003) or by unilateral 

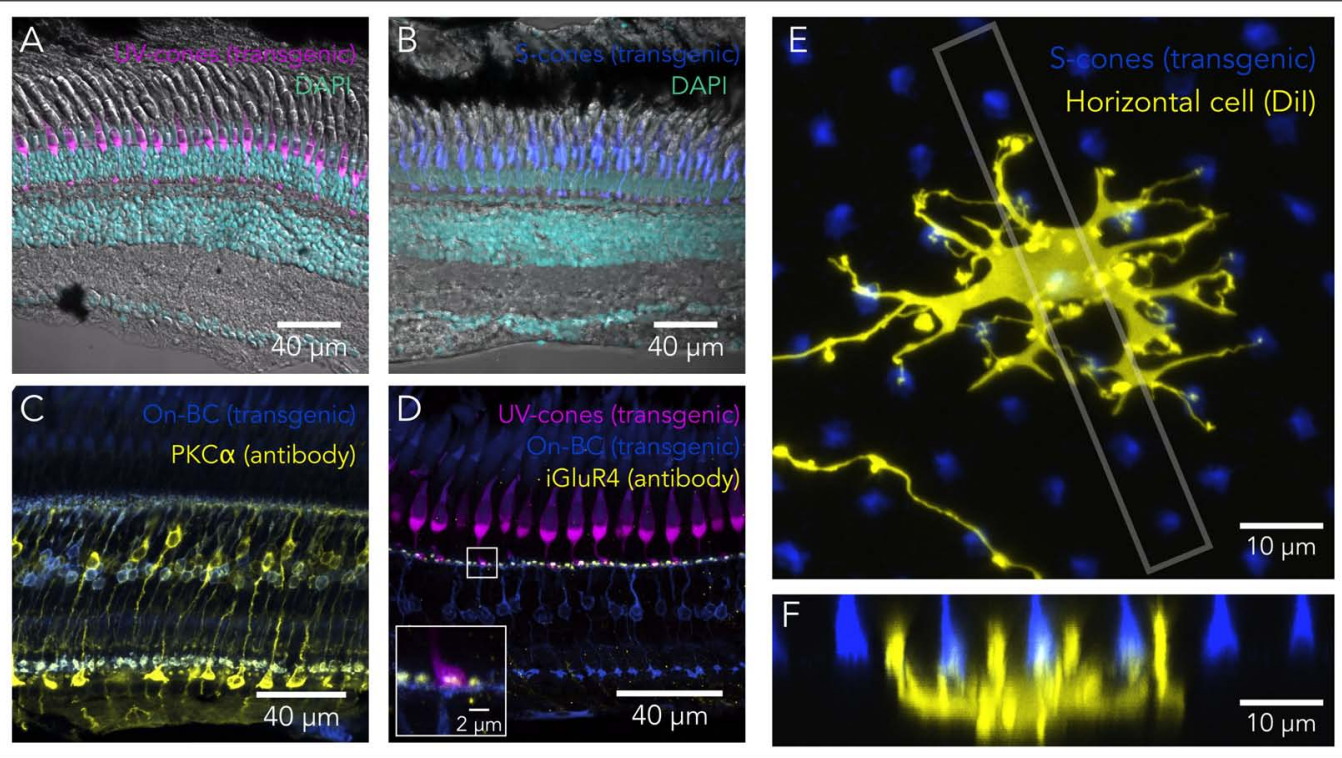

FIGURE 3 | Tools to study retinal circuits. (A) Confocal image of the UV-cone reporter line $T g(s w s \text { 1:GFP) })^{k j 9}$ retina (magenta), overlaid with DAPI nuclear staining (cyan) and a transmitted DIC image. (B) Confocal image of the S-cone reporter line Tg(-3.5opn1sw2:EGFP)k11 retina (blue), overlaid with DAPI nuclear staining (cyan) and a transmitted DIC image. (C) Bipolar cell labeling using PKC $\alpha$ immunolabeling (yellow) and the On-bipolar reporter line Tg(grm6b:EGFP)zh1 (blue).

Rod-contacting bipolar cells are brightly labeled by the PKC $\alpha$ antibody, while another subset of bipolars is more dimly labeled. Some of these are doubly labeled with the transgenic line. (D) Immunolabeling of off-bipolar synapses with photoreceptors using an antibody against the inotropic glutamate receptor type 4 (gria4), in the background of a double transgenic reporter line Tg(sws1:GFP) $)^{k j 9}$ (magenta) and Tg(grm6b:EGFP) zh1 (blue). Inset shows that the punctate labeling in the IPL overlaps with the synaptic terminals of cones and the bipolar cell dendrites. (E) Sparse labeling of horizontal cells using Dil (yellow) in the background of the S-cone reporter line $T g(-3.50 p n 1 s w 2: E G F P)^{k 11}$. Image corresponds to a maximal intensity projection of a confocal stack, where synaptic contacts between the horizontal cell and S-cones are apparent. (F) Maximal intensity projection of an orthogonal view restricted to the box gray in (E), highlighting the invaginations of horizontal processes into the cone synaptic terminals. This particular projection is oriented through a row of UV- and S-cones and the bigger horizontal processes most likely correspond to invaginations into UV-cone terminals.

ocular damage, where recovery of a normal tilt is a sign of functional recovery (Mensinger and Powers, 1999; Lindsey and Powers, 2007; Mensinger and Powers, 2007; Sherpa et al., 2014). In zebrafish, information from the vestibular system is capable of overriding visual input, so that the DLR is only apparent when the vestibular system has been damaged (Nicolson et al., 1998) or when fish are placed head down in a tightly fitting tube (Neuhauss, 2003).

In captivity, zebrafish are conditioned to move toward the front of the tank and wait for food whenever a person approaches. This conditioned learning can be exploited to test for visual function (place preference test) (Sherpa et al., 2014). A very similar test has been recently used in cichlids to demonstrate their ability to truly discriminate colors (Escobar-Camacho et al., 2017) (Table 3).

Interestingly, some of these behaviors seem to rely on only into small subsets of retinal circuits. For example, the OKR seems to be mainly driven by $\mathrm{M}$ - and L-cone signals (Orger and Baier, 2005), while the tracking of small dots, at least in goldfish, depends mainly on M-cone signals (Gehres and Neumeyer, 2007). Similarly, photoavoidance is robustly driven by UV-light, and presumably UV-cone signals (Guggiana-Nilo and Engert, 2016). From previous work that has shown fast functional OKR recovery after $S$-cone ablation even before regeneration and rewiring occur (Hagerman et al., 2016), it is clear that not enough is known about the function, recovery and plasticity of circuits in the retina. Together, behavioral assays along with NTR-mediated ablation of a given cone subtype, could be used to further expand our understanding of these circuits, and the functional consequences of regeneration and rewiring of the different retinal circuits.

\section{Forward and Reverse Genetic Approaches}

Zebrafish forward genetic screens are extremely powerful, and have been successfully used to uncover novel genes that are involved in photoreceptor function and RD (Brockerhoff and Fadool, 2011). It is likely that any additional screening that relies on alterations in visual behaviors will continue to uncover new genes that affect photoreceptor function or cause $\mathrm{RD}$. As more causes of RD continue to be unveiled, we now understand that there are limited number of converging pathways that eventually lead to photoreceptor degeneration, including: classic apoptosis, oxidation, activation of proteolytic pathways, misbalance in intracellular levels of cGMP and calcium, and epigenetic regulation (Trifunovic et al., 2012). With this knowledge, reversegenetic approaches using CRISPR-mediated gene editing to target previously undescribed components within these pathways are likely to be extremely useful in the future. Recent studies in 
TABLE 2 | Toolkit for the study of retinal development and regeneration: antibodies and fluorescent labeling.

\begin{tabular}{|c|c|c|c|c|c|}
\hline $\begin{array}{l}\text { Labeled cell } \\
\text { types or } \\
\text { structures }\end{array}$ & Antibody & Antigen & $\begin{array}{l}\text { Manufacturer (Catalog number) } \\
\text { Species }\end{array}$ & ZFIN ID & Reference \\
\hline \multicolumn{6}{|l|}{ Photoreceptors } \\
\hline Rods & $1 \mathrm{D} 4$ & C-terminus of bovine rhodopsin & $\begin{array}{l}\text { Sigma-Aldrich (MAB5356) Abcam } \\
\text { (ab5417) Mouse monoclonal }\end{array}$ & ZDB-ATB-110114-2 & Linder et al., 2011 \\
\hline Rods & gnat1 & $\begin{array}{l}\text { Human transducin alpha } \\
\text { subunit }\end{array}$ & $\begin{array}{l}\text { ProteinTech (55167-1-AP) Rabbit } \\
\text { polyclonal }\end{array}$ & ZDB-ATB-151005-5 & Liu et al., 2015 \\
\hline \multirow[t]{2}{*}{ Cones } & Recoverin & Human recoverin & Millipore (AB5585) Rabbit polyclonal & ZDB-ATB-151016-2 & Solin et al., 2014 \\
\hline & $\begin{array}{l}\text { Peanut } \\
\text { agglutinin }\end{array}$ & Cone extracellular matrix & Molecular Probes (L21409) & & $\begin{array}{l}\text { Hageman and } \\
\text { Johnson, } 1986\end{array}$ \\
\hline \multirow[t]{2}{*}{ M- and L-cones } & zpr1 & Arrestin3a & $\begin{array}{l}\text { ZIRC (AB_10013803) Mouse } \\
\text { Monoclonal }\end{array}$ & ZDB-ATB-081002-43 & Ile et al., 2010 \\
\hline & $1 \mathrm{D} 4$ & C-terminus of bovine rhodopsin & Abcam (ab5417) Mouse monoclonal & ZDB-ATB-121128-10 & Yin et al., 2012 \\
\hline \multirow[t]{3}{*}{$\begin{array}{l}\text { Photoreceptor } \\
\text { synapses }\end{array}$} & ribeyeA & Ribeye A & $\begin{array}{l}\text { Teresa Nicolson Laboratory OHSU } \\
\text { Rabbit Polyclonal }\end{array}$ & ZDB-ATB-120504-2 & Randlett et al., 2013 \\
\hline & SV2 & Synaptic Vesicle glycoprotein 2 & $\begin{array}{l}\text { DSHB, lowa, United States Mouse } \\
\text { monoclonal }\end{array}$ & ZDB-ATB-081201-1 & Huang et al., 2012 \\
\hline & syntaxin3 & Syntaxin 3 & $\begin{array}{l}\text { Synaptic Systems (110033) Rabbit } \\
\text { polyclonal }\end{array}$ & ZDB-ATB-160428-2 & Lin et al., 2016 \\
\hline \multicolumn{6}{|l|}{ Horizontal cells } \\
\hline & GluR2 & Glutamate receptor type 2 & Millipore (MAB397) Mouse monoclonal & ZDB-ATB-151118-1 & $\begin{array}{l}\text { Yazulla and Studholme, } \\
2001\end{array}$ \\
\hline & GAD67 & $\begin{array}{l}\text { Glutamate decarboxylase } \\
67 \mathrm{kDa} \text { isoform }\end{array}$ & $\begin{array}{l}\text { Chemicon International Inc. (AB108) } \\
\text { Rabbit Polyclonal }\end{array}$ & ZDB-ATB-100903-8 & $\begin{array}{l}\text { Yazulla and Studholme, } \\
2001\end{array}$ \\
\hline \multicolumn{6}{|l|}{ Bipolar cells } \\
\hline $\begin{array}{l}\text { Bipolar cells } \\
\text { (subset) }\end{array}$ & $\mathrm{PKC} \alpha$ & Protein kinase $\mathrm{C}$ alpha subunit & Sigma (P4334) Rabbit polyclonal & ZDB-ATB-090223-3 & $\begin{array}{l}\text { Yazulla and Studholme, } \\
\text { 2001; Moshiri et al., } \\
2008\end{array}$ \\
\hline $\begin{array}{l}\text { On-bipolar cell } \\
\text { subtype }\end{array}$ & mGluR1 & $\begin{array}{l}\text { metabotropic glutamate } \\
\text { receptor } 1 \text { alpha subunit }\end{array}$ & Millipore (AB1551) Rabbit polyclonal & ZDB-ATB-100810-1 & $\begin{array}{l}\text { Yazulla and Studholme, } \\
2001\end{array}$ \\
\hline $\begin{array}{l}\text { On- and Off-bipolar } \\
\text { cells }\end{array}$ & $\begin{array}{l}\text { anti-GABAa } \\
\alpha 3\end{array}$ & GABA receptor alpha subunit 3 & $\begin{array}{l}\text { Alomone Labs (\#AGA-003) Rabbit } \\
\text { polyclonal }\end{array}$ & ZDB-ATB-100903-6 & $\begin{array}{l}\text { Yazulla and Studholme, } \\
2001\end{array}$ \\
\hline On Bipolar Cells & $\begin{array}{l}\text { PKC } \beta 1 \\
(\mathrm{C}-16)\end{array}$ & Protein kinase $\mathrm{C}$ beta subunit 1 & $\begin{array}{l}\text { Santa Cruz Biotechnology, sc-209 } \\
\text { Rabbit polyclonal }\end{array}$ & ZDB-ATB-120614-1 & Glasauer et al., 2016 \\
\hline $\begin{array}{l}\text { On-bipolar cell } \\
\text { dendrites }\end{array}$ & Pan-Maguk & $\begin{array}{l}\text { Membrane-associated } \\
\text { guanylate kinases }\end{array}$ & $\begin{array}{l}\text { Neuromab (75-029, clone K28/86) } \\
\text { Mouse monoclonal }\end{array}$ & ZDB-ATB-120504-1 & Randlett et al., 2013 \\
\hline $\begin{array}{l}\text { Off-bipolar cell } \\
\text { dendrites }\end{array}$ & gria4 & $\begin{array}{l}\text { lonotropic glutamate receptor } \\
\text { type } 4\end{array}$ & Millipore (AB1508) Rabbit polyclonal & ZDB-ATB-100810-2 & $\begin{array}{l}\text { Yazulla and Studholme, } \\
2001\end{array}$ \\
\hline \multicolumn{6}{|l|}{ Amacrine cells } \\
\hline Starburst & ChAT & $\begin{array}{l}\text { Human placental choline } \\
\text { acetyltransferase }\end{array}$ & Millipore, AB144P, Goat polyclonal & ZDB-ATB-081017-3 & Glasauer et al., 2016 \\
\hline GABAergic & GAD65/67 & $\begin{array}{l}\text { Glutamic acid decarboxylase } \\
65 \mathrm{kDa} / 67 \mathrm{kDa}\end{array}$ & Abcam (Ab11070) Rabbit polyclonal & ZDB-ATB-090617-2 & Glasauer et al., 2016 \\
\hline Dopaminergic & $\mathrm{TH}$ & Tyrosine hydroxylase & $\begin{array}{l}\text { Immunostar, Inc. (22941) Mouse } \\
\text { monoclonal }\end{array}$ & ZDB-ATB-081017-8 & Glasauer et al., 2016 \\
\hline \multicolumn{6}{|l|}{ Müller glia } \\
\hline & GS & Glutamine Synthase & Millipore (mab302) Mouse monoclonal & ZDB-ATB-081009-5 & Randlett et al., 2013 \\
\hline & gfap & Glial Fibrillary Acidic Protein & ZIRC (zrf-1) Mouse monoclonal & ZDB-ATB-081002-46 & Solin et al., 2014 \\
\hline & glt1 & Glutamate transporter 1 (glial) & $\begin{array}{l}\text { Millipore (AB1783) Guinea pig } \\
\text { polyclonal }\end{array}$ & ZDB-ATB-100916-6 & $\begin{array}{l}\text { Yazulla and Studholme, } \\
2001\end{array}$ \\
\hline $\begin{array}{l}\text { Ciliary Marginal } \\
\text { Zone }\end{array}$ & PCNA & $\begin{array}{l}\text { Anti-Proliferating Cell Nuclear } \\
\text { Antigen }\end{array}$ & $\begin{array}{l}\text { Santa Cruz Biotechnology, sc-56 } \\
\text { Mouse monoclonal }\end{array}$ & ZDB-ATB-081121-4 & $\begin{array}{l}\text { Inoue and Wittbrodt, } \\
2011\end{array}$ \\
\hline $\begin{array}{l}\text { Bipolar, horizontal } \\
\text { and amacrine cells }\end{array}$ & isl1 & Islet 1 & $\begin{array}{l}\text { DSHB, lowa, United States (40.3A4) } \\
\text { Mouse monoclonal }\end{array}$ & ZDB-ATB-081124-3 & Zhang et al., 2012 \\
\hline $\begin{array}{l}\text { Bipolar, horizontal, } \\
\text { amacrine and }\end{array}$ & $\mathrm{HuC} / \mathrm{HuD}$ & $\begin{array}{l}\text { ELAV like neuron-specific RNA } \\
\text { binding protein } 3 \text { and } 4\end{array}$ & $\begin{array}{l}\text { Invitrogen (A-21271) Mouse } \\
\text { monoclonal }\end{array}$ & ZDB-ATB-081003-2 & Randlett et al., 2013 \\
\hline
\end{tabular}

ganglion cells 
TABLE 3 | Toolkit for the study of retinal development and regeneration: electroretinograms and visually guided behaviors.

\begin{tabular}{|c|c|c|}
\hline Visual assay & Description & References \\
\hline Electroretinogram (ERG) & $\begin{array}{l}\text { Measurement of the changes in the bulk electrical activity produced by the } \\
\text { retina when stimulated with light. Can be measured in both larvae and adults. }\end{array}$ & Hughes et al., 1998; Chrispell et al., 2015 \\
\hline Optokinetic reflex (OKR) & $\begin{array}{l}\text { Rotational eye movements track moving targets, usually white and black stripes } \\
\text { in a rotating drum, are followed by saccades that reset the eye position. }\end{array}$ & $\begin{array}{l}\text { Clark, 1981; Neuhauss et al., 1999; Neuhauss, } \\
2003\end{array}$ \\
\hline Optomotor response (OMR) & Swimming in the direction of a moving stimuli. & Clark, 1981; Neuhauss, 2003 \\
\hline Prey/Small object tracking & $\begin{array}{l}\text { Larvae hunt for paramecia by making stereotyped swimming movements to } \\
\text { orient themselves before swimming forward to capture their prey }\end{array}$ & $\begin{array}{l}\text { Borla et al., 2002; Gahtan et al., 2005; } \\
\text { McElligott and O'Malley, } 2005\end{array}$ \\
\hline Phototaxis and Photoavoidance & Swim movements towards or away from light sources & $\begin{array}{l}\text { Brockerhoff et al., } 1995 \text { Orger and Baier, 2005; } \\
\text { Burgess et al., } 2010\end{array}$ \\
\hline $\begin{array}{l}\text { Escape response to sudden } \\
\text { decreases in illumination }\end{array}$ & $\begin{array}{l}\text { Larval zebrafish exhibit escape responses ("O-bends") to sudden decreases in } \\
\text { illumination }\end{array}$ & Miller et al., 2018 \\
\hline $\begin{array}{l}\text { Escape response to dark } \\
\text { objects }\end{array}$ & $\begin{array}{l}\text { Adult zebrafish avoid moving dark objects by abruptly changing the direction of } \\
\text { swimming }\end{array}$ & Li and Dowling, 1997 \\
\hline Dorsal light reflex (DLR) & $\begin{array}{l}\text { Zebrafish tilt their bodies to turn their backs towards the source of illumination } \\
\text { by keeping equal input in both eyes, but vestibular information can override this } \\
\text { reflex. Reflex can be made apparent by placing fish head down in a tightly fitting } \\
\text { tube. }\end{array}$ & Nicolson et al., 1998; Neuhauss, 2003 \\
\hline Place preference & $\begin{array}{l}\text { Captive zebrafish are conditioned by feeding routines to move toward front of } \\
\text { the tank when they visually detect a person approaching. }\end{array}$ & Sherpa et al., 2014 \\
\hline
\end{tabular}

zebrafish have already demonstrated that zebrafish can be used as a platform to rapidly perform genetic screens using CRISPR (Varshney et al., 2015, 2016; Shah et al., 2016). In addition to genetic screens, similar to what has been done in fin and hair-cell regeneration studies (Mathew et al., 2007; Namdaran et al., 2012), pharmacology-based screening could be used to isolate novel compounds with the ability to promote or prevent photoreceptor regeneration. Similar screens could also be accomplished using behavioral assays, evaluating the recovery of visual function after regeneration. Some of the success of such screens in hair cells of the lateral line stems from the fast regeneration times ( $\sim 2$ days) and the small number of cell types that have to be regenerated. In paradigms of regeneration after extensive damage (surgical of pharmacological) in adults, the long regeneration times (8-14 weeks) and the diversity of cell types that need to be regenerated might present insurmountable hurdles for screens. Nevertheless, photoreceptor regeneration occurs within 4 weeks in adults and in $\sim 10$ days in larvae after cell-specific ablations, opening up the possibility to carry out such screens.

While the majority of regeneration studies in zebrafish have focused on regenerating damaged cells, for functional recovery after regeneration, it is imperative that new cells integrate appropriately into their specific retinal circuits. Our current knowledge on how retinal circuits in the outer retina form during development and after regenerations is limited. Studies into these processes suggest that rewiring after regeneration is not a complete recapitulation of development. First of all, during development retinal cells are derived from retinal progenitors and retinal circuits assemble properly even in the absence of Müller glia (Williams et al., 2010). In contrast, during regeneration, Müller glia are the principal source of new retinal cells. Second, not all the transcription pathways that are active during development are reactivated during regeneration (Veldman et al., 2007; Sherpa et al., 2014). Third, there seems to be more plasticity and a greater capacity for compensation.
It appears that during rewiring, at least in larvae, maintaining inputs and outputs is more important than the absolute selectivity of connections (D’Orazi et al., 2016; Yoshimatsu et al., 2016). In adult zebrafish, after pharmacological ablation of bipolar, amacrine and ganglion cells (but survival of photoreceptors) and their subsequent regeneration, various subtypes of BCs seem to be able to recapitulate the diversity of connectivity that is found in uninjured eyes, but as a population, selectivity for photoreceptors seems to be restored (McGinn et al., 2018). Further investigation is required to explain the disagreement between these studies since there are many differences including ablation technique (NTR-MTZ vs. ouabain), age of ablation (larvae vs. adults), time between ablation and assessment of connectivity (1-2 weeks vs. 8 weeks) and subtypes of cells studied. Yet, this raises interesting questions: is the capacity for compensation only present in larvae and lost in the adult? or, is compensation only present in the initial phase after regeneration and normal selectivity of connections reestablished over time?

It is interesting to note that selectivity is not lost during rewiring, it is just more permissive, and it is very likely that the same molecules that allow recognition between retinal cell types are used in both development and regeneration. Only a handful of cell-adhesion and synaptic molecules are known to be necessary for the formation of synaptic contacts between photoreceptors and downstream retinal cells (Zhang et al., 2017; Miller et al., 2018). Some of these molecules are key across all photoreceptors, while others are specific to rods (Cao et al., 2015; Wang et al., 2017) or to cones (Sarria et al., 2018; Ueno et al., 2018). To date, the molecular mechanisms involved in the recognition between specific photoreceptor subtypes and their synaptic partners (horizontal and bipolar cells) are not known. Any of the genetic screening tools mentioned above could be combined with the NTR-MTZ transgenic lines (Table 1), to target specific cell subtypes and elucidate the mechanisms that enable rewiring. Research in this front could have a very 
significant impact in phenotyping vision loss in $\mathrm{RD}$, and to develop manipulations that could ultimately enable rewiring of transplanted or regenerated photoreceptors into proper retinal circuits.

\section{Gene-Expression Profiling (RNAseq)}

During the last decade, advances in the capacity of highthroughput sequencing has allowed to profile the transcriptomes of whole tissues or dissociated single cells. Gene-expression profiling of retinal cells in mice has given great insight in the classification of retinal cells into different (and even novel) subtypes (Macosko et al., 2015), especially for BC, where clear differences in molecules involved in cell-recognition and synapse formation were detected (Shekhar et al., 2016). These techniques can be applied in zebrafish, especially using transgenic lines, as has been recently reported for rods (Sun et al., 2018). RNAseq of zebrafish retinal cells could help unveil the genes that are required for synapse formation between $\mathrm{BC}$ and photoreceptors, genes that could be essential to promote rewiring in $\mathrm{RD}$ therapies. RNAseq could also be exploited to study the changes in gene expression that occur during degeneration and regeneration. This could be accomplished by profiling single cells in the most relevant time points after photoreceptor death. With a focus on Müller glia, further insights could be gained into the gene networks that allow pluripotent and functional recovery in zebrafish. This knowledge will also be extremely valuable for the treatment of RD and understanding how to initiate regeneration after RD.

\section{CONCLUSION AND OUTLOOK}

Our field is developing a deep understanding on many aspects of $\mathrm{RD}$, including risk factors, underlying genetic causes, molecular pathways that lead to photoreceptor death, and the manipulations that could slow down the progression of the disease. During the last decade, we have made significant progress into revolutionary therapies that could, 1 day, cure blindness.

Despite all of the research on $\mathrm{RD}$ and regeneration, there are still gaps in our current knowledge that limit our capacity to understand certain aspects of RD and hinder our ability to develop therapies. The zebrafish is an advantageous model to fill in these gaps, especially at a mechanistic level. As a relevant example, we have discussed how zebrafish has been

\section{REFERENCES}

Adler, R. (2008). Curing blindness with stem cells: hope, reality, and challenges. Adv. Exp. Med. Biol. 613, 3-20. doi: 10.1007/978-0-387-74904-4_1

Antinucci, P., and Hindges, R. (2016). A crystal-clear zebrafish for in vivo imaging. Sci. Rep. 6:29490. doi: 10.1038/srep29490

Ariga, J., Walker, S. L., and Mumm, J. S. (2010). Multicolor time-lapse imaging of transgenic zebrafish: visualizing retinal stem cells activated by targeted neuronal cell ablation. J. Vis. Exp. 43:2093. doi: 10.3791/2093

Barber, A. C., Hippert, C., Duran, Y., West, E. L., Bainbridge, J. W., WarreCornish, K., et al. (2013). Repair of the degenerate retina by photoreceptor transplantation. Proc. Natl. Acad. Sci. U.S.A. 110, 354-359. doi: 10.1073/pnas. 1212677110 used to delineate molecular pathways within Müller glia that allow regeneration of retinal cells even in the adult zebrafish. This knowledge has been directly applied into the mouse retina and successfully used to generate new and functional bipolar and amacrine cells (Jorstad et al., 2017). Although studies have been able to stimulate retinal regeneration in mice using manipulations derived from the study of zebrafish retinal regeneration, but we do not yet fully understand the pathways required to regenerate each retinal cell type, or how these pathways are regulated to regenerate specific subpopulations. Further studies are required to understand the mechanisms that allow zebrafish Müller glia to not only produce any retinal cell, but also to specifically replace the lost population without overtly producing proliferation of undamaged cell types (D'Orazi et al., 2016; Yoshimatsu et al., 2016; McGinn et al., 2018).

In addition to gaps in knowledge on the role of Müller glia and regeneration, we also have an incomplete grasp on the processes that are involved in recognition between photoreceptors and their postsynaptic targets, with only a handful of molecules known to be involved in the correct formation of synapses. This leaves us with little leverage on manipulations that could promote integration of new photoreceptors into the surviving retinal circuits. Solving these issues and finding viable therapeutic options for RD will certainly require diverse approaches. Research in zebrafish is uniquely poised to make additional key contributions into $\mathrm{RD}$, especially on unveiling the molecular mechanisms involved in photoreceptor regeneration and the processes that guide wiring during development and rewiring after regeneration of photoreceptors into retinal circuits.

\section{AUTHOR CONTRIBUTIONS}

JA conceived and carried out the literature review research, designed the figures and diagrams, acquired the images, and wrote the article. KK conceived and carried out the literature review research and wrote the article.

\section{FUNDING}

This work was supported by NIH/NIDCD intramural research funds 1ZIADC000085-01 (KK), and NIH/NEI intramural research funds ZIAEY000488-10 (JA).

Bernardos, R. L., Barthel, L. K., Meyers, J. R., and Raymond, P. A. (2007). Late-stage neuronal progenitors in the retina are radial Müller glia that function as retinal stem cells. J. Neurosci. 27, 7028-7040. doi: 10.1523/JNEUROSCI.1624-07. 2007

Bibliowicz, J., Tittle, R. K., and Gross, J. M. (2011). Toward a better understanding of human eye disease insights from the zebrafish, danio rerio. Prog. Mol. Biol. Transl. Sci. 100, 287-330. doi: 10.1016/B978-0-12-384878-9.00007-8

Borla, M. A., Palecek, B., Budick, S., and O'Malley, D. M. (2002). Prey capture by larval zebrafish: evidence for fine axial motor control. Brain Behav. Evol. 60, 207-229. doi: 10.1159/000066699

Bourne, R. R. A., Flaxman, S. R., Braithwaite, T., Cicinelli, M. V., Das, A., Jonas, J. B., et al. (2017). Magnitude, temporal trends, and projections of the global prevalence of blindness and distance and near vision impairment: a systematic 
review and meta-analysis. Lancet Glob. Health 5, e888-e897. doi: 10.1016/ S2214-109X(17)30293-0

Bringmann, A., Pannicke, T., Biedermann, B., Francke, M., Iandiev, I., Grosche, J., et al. (2009). Role of retinal glial cells in neurotransmitter uptake and metabolism. Neurochem. Int. 54, 143-160. doi: 10.1016/j.neuint.2008.10.014

Brockerhoff, S. E., and Fadool, J. M. (2011). Genetics of photoreceptor degeneration and regeneration in zebrafish. Cell. Mol. Life Sci. 68, 651-659. doi: 10.1007/ s00018-010-0563-8

Brockerhoff, S. E., Hurley, J. B., Janssen-Bienhold, U., Neuhauss, S. C., Driever, W. Dowling, J. E., et al. (1995). A behavioral screen for isolating zebrafish mutants with visual system defects. Proc. Natl. Acad. Sci. U.S.A. 92, 10545-10549. doi: 10.1073/pnas.92.23.10545

Burgess, H. A., and Granato, M. (2007). Modulation of locomotor activity in larval zebrafish during light adaptation. J. Exp. Biol. 210(Pt 14), 2526-2539. doi: 10.1242/jeb.003939

Burgess, H. A., Schoch, H., and Granato, M. (2010). Distinct retinal pathways drive spatial orientation behaviors in zebrafish navigation. Curr. Biol. 20, 381-386. doi: 10.1016/j.cub.2010.01.022

Busskamp, V., Duebel, J., Balya, D., Fradot, M., Viney, T. J., Siegert, S., et al. (2010). Genetic reactivation of cone photoreceptors restores visual responses in retinitis pigmentosa. Science 329, 413-417. doi: 10.1126/science.1190897

Cameron, D. A. (2000). Cellular proliferation and neurogenesis in the injured retina of adult zebrafish. Vis. Neurosci. 17, 789-797. doi: 10.1017/ S0952523800175121

Cao, Y., Sarria, I., Fehlhaber, K. E., Kamasawa, N., Orlandi, C., James, K. N., et al. (2015). Mechanism for selective synaptic wiring of rod photoreceptors into the retinal circuitry and its role in vision. Neuron 87, 1248-1260. doi: 10.1016/j.neuron.2015.09.002

Carter-Dawson Louvenia, D., and Lavail Matthew, M. (1979). Rods and cones in the mouse retina. I. Structural analysis using light and electron microscopy. J. Compar. Neurol. 188, 245-262. doi: 10.1002/cne.901880204

Centanin, L., Hoeckendorf, B., and Wittbrodt, J. (2011). Fate restriction and multipotency in retinal stem cells. Cell Stem Cell 9, 553-562. doi: 10.1016/j.stem. 2011.11.004

Chang, B., Grau, T., Dangel, S., Hurd, R., Jurklies, B., Sener, E. C., et al. (2009). A homologous genetic basis of the murine cpfl1 mutant and human achromatopsia linked to mutations in the pde6c gene. Proc. Natl. Acad. Sci. U.S.A. 106, 19581-19586. doi: 10.1073/pnas.0907720106

Chinen, A., Hamaoka, T., Yamada, Y., and Kawamura, S. (2003). Gene duplication and spectral diversification of cone visual pigments of zebrafish. Genetics 163, 663-675.

Chrispell, J. D., Rebrik, T. I., and Weiss, E. R. (2015). Electroretinogram analysis of the visual response in zebrafish larvae. J. Vis. Exp. 97:52662. doi: 10.3791/52662

Cideciyan, A. V. (2010). Leber congenital amaurosis due to rpe65 mutations and its treatment with gene therapy. Prog. Retin. Eye Res. 29, 398-427. doi: 10.1016/ j.preteyeres.2010.04.002

Clark, D. T. (1981). Visual Responses in the Developing Zebrafish (Brachydanio rerio). Ph.D. thesis, Eugene, OR, University of Oregon.

Connaughton, V. P., Graham, D., and Nelson, R. (2004). Identification and morphological classification of horizontal, bipolar, and amacrine cells within the zebrafish retina. J. Comp. Neurol. 477, 371-385. doi: 10.1002/cne.20261

da Cruz, L., Dorn, J. D., Humayun, M. S., Dagnelie, G., Handa, J., Barale, P. O., et al. (2016). Five-year safety and performance results from the argus ii retinal prosthesis system clinical trial. Ophthalmology 123, 2248-2254. doi: 10.1016/j. ophtha.2016.06.049

Daiger, S. P., Sullivan, L. S., and Bowne, S. J. (2013). Genes and mutations causing retinitis pigmentosa. Clin. Genet. 84, 132-141. doi: 10.1111/cge.12203

Daniele, L. L., Emran, F., Lobo, G. P., Gaivin, R. J., and Perkins, B. D. (2016). Mutation of wrb, a component of the guided entry of tail-anchored protein pathway, disrupts photoreceptor synapse structure and function. Invest. Ophthalmol. Vis. Sci. 57, 2942-2954. doi: 10.1167/iovs.15-18996

De Bode, C. (2017). Blindness and Visual Impairment. Available at: http://www. who.int/news-room/fact-sheets/detail/blindness-and-visual-impairment [accessed May 28, 2018].

D’Orazi, F. D., Zhao, X. F., Wong, R. O., and Yoshimatsu, T. (2016). Mismatch of synaptic patterns between neurons produced in regeneration and during development of the vertebrate retina. Curr. Biol. 26, 2268-2279. doi: 10.1016/ j.cub.2016.06.063
Dreosti, E., Odermatt, B., Dorostkar, M. M., and Lagnado, L. (2009). A genetically encoded reporter of synaptic activity in vivo. Nat. Methods 6, 883-889. doi: 10.1038/nmeth.1399

Duval, M. G., Chung, H., Lehmann, O. J., and Allison, W. T. (2013). Longitudinal fluorescent observation of retinal degeneration and regeneration in zebrafish using fundus lens imaging. Mol. Vis. 19, 1082-1095.

Dyer, M. A., and Cepko, C. L. (2000). Control of Müller glial cell proliferation and activation following retinal injury. Nat. Neurosci. 3, 873-880. doi: 10.1038/ 78774

Ellett, F., Pase, L., Hayman, J. W., Andrianopoulos, A., and Lieschke, G. J. (2011). Mpeg1 promoter transgenes direct macrophage-lineage expression in zebrafish. Blood 117, e49-e56. doi: 10.1182/blood-2010-10-314120

Escobar-Camacho, D., Marshall, J., and Carleton, K. L. (2017). Behavioral color vision in a cichlid fish: Metriaclima benetos. J. Exp. Biol. 220, 2887-2899. doi: $10.1242 /$ jeb. 160473

Fadool, J. M. (2003). Rod genesis in the teleost retina as a model of neural stem cells. Exp. Neurol. 184, 14-19. doi: 10.1016/S0014-4886(03)00309-1

Farrar, G. J., Carrigan, M., Dockery, A., Millington-Ward, S., Palfi, A. Chadderton, N., et al. (2017). Toward an elucidation of the molecular genetics of inherited retinal degenerations. Hum. Mol. Genet. 26, R2-R11. doi: 10.1093/ hmg/ddx185

Fausett, B. V., and Goldman, D. (2006). A role for $\alpha 1$ tubulin-expressing Müller glia in regeneration of the injured zebrafish retina. J. Neurosci. 26, 6303-6313. doi: 10.1523/JNEUROSCI.0332-06.2006

Fausett, B. V., Gumerson, J. D., and Goldman, D. (2008). The proneural basic helix-loop-helix gene asclla is required for retina regeneration. J. Neurosci. 28, 1109-1117. doi: 10.1523/JNEUROSCI.4853-07.2008

Fimbel, S. M., Montgomery, J. E., Burket, C. T., and Hyde, D. R. (2007). Regeneration of inner retinal neurons after intravitreal injection of ouabain in zebrafish. J. Neurosci. 27, 1712-1724. doi: 10.1523/JNEUROSCI.5317-06.2007

Förster, D., Dal Maschio, M., Laurell, E., and Baier, H. (2017). An optogenetic toolbox for unbiased discovery of functionally connected cells in neural circuits. Nat. Commun. 8:116. doi: 10.1038/s41467-017-00160-z

Fraser, B., DuVal, M. G., Wang, H., and Allison, W. T. (2013). Regeneration of cone photoreceptors when cell ablation is primarily restricted to a particular cone subtype. PLoS One 8:e55410. doi: 10.1371/journal.pone.0055410

Gahtan, E., Tanger, P., and Baier, H. (2005). Visual prey capture in larval zebrafish is controlled by identified reticulospinal neurons downstream of the tectum. J. Neurosci. 25, 9294-9303. doi: 10.1523/JNEUROSCI.2678-05.2005

Ganzen, L., Venkatraman, P., Pang, C. P., Leung, Y. F., and Zhang, M. (2017). Utilizing zebrafish visual behaviors in drug screening for retinal degeneration. Int. J. Mol. Sci. 18:E1185. doi: 10.3390/ijms18061185

Gehres, M., and Neumeyer, C. (2007). Small field motion detection in goldfish is red-green color blind and mediated by the m-cone type. Vis. Neurosci. 24, 399-407. doi: 10.1017/S0952523807070447

Glasauer, S. M., Wager, R., Gesemann, M., and Neuhauss, S. C. (2016). Mglur6b:Egfp transgenic zebrafish suggest novel functions of metabotropic glutamate signaling in retina and other brain regions. J. Comp. Neurol. 524, 2363-2378. doi: 10.1002/cne.24029

Gollisch, T., and Meister, M. (2010). Eye smarter than scientists believed: neural computations in circuits of the retina. Neuron 65, 150-164. doi: 10.1016/j. neuron.2009.12.009

Guggiana-Nilo, D. A., and Engert, F. (2016). Properties of the visible light phototaxis and uv avoidance behaviors in the larval zebrafish. Front. Behav. Neurosci. 10:160. doi: 10.3389/fnbeh.2016.00160

Hageman, G. S., and Johnson, L. V. (1986). Biochemical characterization of the major peanut-agglutinin-binding glycoproteins in vertebrate retinae. J. Comp. Neurol. 249, 499-510,482-493. doi: 10.1002/cne.902490406

Hagerman, G. F., Noel, N. C., Cao, S. Y., DuVal, M. G., Oel, A. P., and Allison, W. T. (2016). Rapid recovery of visual function associated with blue cone ablation in zebrafish. PLoS One 11:e0166932. doi: 10.1371/journal.pone.016 6932

Hamaoka, T., Takechi, M., Chinen, A., Nishiwaki, Y., and Kawamura, S. (2002). Visualization of rod photoreceptor development using gfp-transgenic zebrafish. Genesis 34, 215-220. doi: 10.1002/gene.10155

Hitchcock, P. F., and Cirenza, P. (1994). Synaptic organization of regenerated retina in the goldfish. J. Compar. Neurol. 343, 609-616. doi: 10.1002/cne. 903430410 
Hitchcock, P. F., Lindsey Myhr, K. J., Easter, S. S. Jr., Mangione-Smith, R., and Jones, D. D. (1992). Local regeneration in the retina of the goldfish. J. Neurobiol. 23, 187-203. doi: 10.1002/neu.480230209

Homma, K., Okamoto, S., Mandai, M., Gotoh, N., Rajasimha, H. K., and Chang, Y. S. (2013). Developing rods transplanted into the degenerating retina of crxknockout mice exhibit neural activity similar to native photoreceptors. Stem Cells 31, 1149-1159. doi: 10.1002/stem.1372

Huang, Y. Y., Haug, M. F., Gesemann, M., and Neuhauss, S. C. (2012). Novel expression patterns of metabotropic glutamate receptor 6 in the zebrafish nervous system. PLoS One 7:e35256. doi: 10.1371/journal.pone.0035256

Hughes, A., Saszik, S., Bilotta, J., Demarco, P. J. Jr., and Patterson, W. F. (1998). Cone contributions to the photopic spectral sensitivity of the zebrafish erg. Vis. Neurosci. 15, 1029-1037. doi: 10.1017/S095252389815602X

Ile, K. E., Kassen, S., Cao, C., Vihtehlic, T., Shah, S. D., and Mousley, C. J. (2010). Zebrafish class 1 phosphatidylinositol transfer proteins: Pitp $\beta$ and double cone cell outer segment integrity in retina. Traffic 11, 1151-1167. doi: 10.1111/j.16000854.2010.01085.x

Inoue, D., and Wittbrodt, J. (2011). One for all-a highly efficient and versatile method for fluorescent immunostaining in fish embryos. PLoS One 6:e19713. doi: 10.1371/journal.pone.0019713

Jacobson, S. G., Cideciyan, A. V., Ratnakaram, R., Heon, E., Schwartz, S. B., Roman, A. J., et al. (2012). Gene therapy for leber congenital amaurosis caused by rpe65 mutations: safety and efficacy in fifteen children and adults followed up to three years. Arch. Ophthalmol. 130, 9-24. doi: 10.1001/archophthalmol.2011.298

Jacobson, S. G., Cideciyan, A. V., Roman, A. J., Sumaroka, A., Schwartz, S. B., Heon, E., et al. (2015). Improvement and decline in vision with gene therapy in childhood blindness. N. Engl. J. Med. 372, 1920-1926. doi: 10.1056/ NEJMoa1412965

Jager, R. D., Mieler, W. F., and Miller, J. W. (2008). Age-related macular degeneration. N. Engl. J. Med. 358, 2606-2617. doi: 10.1056/NEJMra0801537

Johns, P. R., and Fernald, R. D. (1981). Genesis of rods in teleost fish retina. Nature 293, 141-142. doi: $10.1038 / 293141 \mathrm{a} 0$

Johnston, J., Ding, H., Seibel, S. H., Esposti, F., and Lagnado, L. (2014). Rapid mapping of visual receptive fields by filtered back projection: application to multi-neuronal electrophysiology and imaging. J. Physiol. 592, 4839-4854. doi: 10.1113/jphysiol.2014.276642

Jorstad, N. L., Wilken, M. S., Grimes, W. N., Wohl, S. G., VandenBosch, L. S., Yoshimatsu, T. A., et al. (2017). Stimulation of functional neuronal regeneration from Müller glia in adult mice. Nature 548, 103-107. doi: 10.1038/nature23283

Julian, D., Ennis, K., and Korenbrot, J. I. (1998). Birth and fate of proliferative cells in the inner nuclear layer of the mature fish retina. J. Compar. Neurol. 394, 271-282. doi: 10.1002/(SICI)1096-9861(19980511)394:3<271::AID-CNE1>3.0. $\mathrm{CO} ; 2-\mathrm{Z}$

Karl, M. O., Hayes, S., Nelson, B. R., Tan, K., Buckingham, B., Reh, T. A., et al. (2008). Stimulation of neural regeneration in the mouse retina. Proc. Natl. Acad. Sci. U.S.A. 105, 19508-19513. doi: 10.1073/pnas. 0807453105

Kassen, S. C., Ramanan, V., Montgomery, J. E., T Burket, C., Liu, C. G., Vihtelic, T. S., et al. (2007). Time course analysis of gene expression during light-induced photoreceptor cell death and regeneration in albino zebrafish. Dev. Neurobiol. 67, 1009-1031. doi: 10.1002/dneu.20362

Klaassen, L. J., de Graaff, W., van Asselt, J. B., Klooster, J., and Kamermans, M. (2016). Specific connectivity between photoreceptors and horizontal cells in the zebrafish retina. J. Neurophysiol. 116, 2799-2814. doi: 10.1152/jn.00449.2016

Korenbrot, J. I., Mehta, M., Tserentsoodol, N., Postlethwait, J. H., and Rebrik, T. I. (2013). Emll (cng-modulin) controls light sensitivity in darkness and under continuous illumination in zebrafish retinal cone photoreceptors. J. Neurosci. 33, 17763-17776. doi: 10.1523/JNEUROSCI.2659-13.2013

Kumaran, N., Moore, A. T., Welebe, R. G., and Michaelides, M. (2017). Leber congenital amaurosis/early-onset severe retinal dystrophy: clinical features, molecular genetics and therapeutic interventions. Br. J. Ophthalmol. 101, 11471154. doi: 10.1136/bjophthalmol-2016-309975

Lazic, R., and Gabric, N. (2007). Verteporfin therapy and intravitreal bevacizumab combined and alone in choroidal neovascularization due to age-related macular degeneration. Ophthalmology 114, 1179-1185. doi: 10.1016/j.ophtha.2007. 03.006

Lewis, P. M., Ayton, L. N., Guymer, R. H., Lowery, A. J., Blamey, P. J., Allen, P. J., et al. (2016). Advances in implantable bionic devices for blindness: a review. ANZ J. Surg. 86, 654-659. doi: 10.1111/ans.13616
Li, L., and Dowling, J. E. (1997). A dominant form of inherited retinal degeneration caused by a non-photoreceptor cell-specific mutation. Proc. Natl. Acad. Sci. U.S.A. 94, 11645-11650. doi: 10.1073/pnas.94.21.11645

Li, L., and Dowling, J. E. (2000). Disruption of the olfactoretinal centrifugal pathway may relate to the visual system defect in night blindness $b$ mutant zebrafish. J. Neurosci. 20, 1883-1892. doi: 10.1523/JNEUROSCI.20-05-01883. 2000

Li, Y. N., Matsui, J. I., and Dowling, J. E. (2009). Specificity of the horizontal cell-photoreceptor connections in the zebrafish (danio rerio) retina. J. Comp. Neurol. 516, 442-453. doi: 10.1002/cne.22135

Li, Y. N., Tsujimura, T., Kawamura, S., and Dowling, J. E. (2012). Bipolar cellphotoreceptor connectivity in the zebrafish (danio rerio) retina. J. Comp. Neurol. 520, 3786-3802. doi: 10.1002/cne.23168

Lin, S. Y., Vollrath, M. A., Mangosing, S., Shen, J., Cardenas, E., and Corey, D. P. (2016). The zebrafish pinball wizard gene encodes wrb, a tail-anchored-protein receptor essential for inner-ear hair cells and retinal photoreceptors. J. Physiol. 594, 895-914. doi: 10.1113/JP271437

Linder, B., Dill, H., Hirmer, A., Brocher, J., Lee, G. P., Mathavan, S., et al. (2011). Systemic splicing factor deficiency causes tissue-specific defects: a zebrafish model for retinitis pigmentosa†. Hum. Mol. Genet. 20, 368-377. doi: 10.1093/ $\mathrm{hmg} / \mathrm{ddq} 473$

Lindsey, A. E., and Powers, M. K. (2007). Visual behavior of adult goldfish with regenerating retina. Vis. Neurosci. 24, 247-255. doi: $10.1017 / \mathrm{S} 0952523806230207$

Liu, F., Chen, J., Yu, S., Raghupathy, R. K., Liu, X., and Qin, Y. (2015). Knockout of rp2 decreases grk1 and rod transducin subunits and leads to photoreceptor degeneration in zebrafish. Hum. Mol. Genet. 24, 4648-4659. doi: 10.1093/hmg/ ddv197

Lombardo, F. (1968). La rigenerazione della retina negli adulti di un teleosteo. Accad. Lincei Rend. Commun. Sci. Fish. Mat. Nat. 45, 631-635.

Lust, K., and Wittbrodt, J. (2018). Activating the regenerative potential of Müller glia cells in a regeneration-deficient retina. elife 7:e32319. doi: 10.7554/eLife. 32319

Lv, C., Gould, T. J., Bewersdorf, J., and Zenisek, D. (2012). High-resolution optical imaging of zebrafish larval ribbon synapse protein ribeye, rim2, and cav 1.4 by stimulation emission depletion microscopy. Microsc. Microanal. 18, 745-752. doi: $10.1017 / S 1431927612000268$

MacDonald, R. B., Kashikar, N. D., Lagnado, L., and Harris, W. A. (2017). A novel tool to measure extracellular glutamate in the zebrafish nervous system in vivo. Zebrafish 14, 284-286. doi: 10.1089/zeb.2016.1385

MacLaren, R. E., Bennett, J., and Schwartz, S. D. (2016). Gene therapy and stem cell transplantation in retinal disease: the new frontier. Ophthalmology 123 , S98-S106. doi: 10.1016/j.ophtha.2016.06.041

Macosko, E. Z., Basu, A., Satija, R., Nemesh, J., Shekhar, K., Goldman, M., et al. (2015). Highly parallel genome-wide expression profiling of individual cells using nanoliter droplets. Cell 161, 1202-1214. doi: 10.1016/j.cell.2015. 05.002

Marvin, J. S., Borghuis, B. G., Tian, L., Cichon, J., Harnett, M. T., Akerboom, J., et al. (2013). An optimized fluorescent probe for visualizing glutamate neurotransmission. Nat. Methods 10, 162-170. doi: 10.1038/nmeth.2333

Mathew, L. K., Sengupta, S., Kawakami, A., Andreasen, E. A., Löhr, C. V., Loynes, C. A., et al. (2007). Unraveling tissue regeneration pathways using chemical genetics. J. Biol. Chem. 282, 35202-35210. doi: 10.1074/jbc.M7066 40200

McElligott, M. B., and O’Malley, D. M. (2005). Prey tracking by larval zebrafish: axial kinematics and visual control. Brain Behav. Evol. 66, 177-196. doi: 10. $1159 / 000087158$

McGinn, T. E., Mitchell, D. M., Meighan, P. C., Partington, N., Leoni, D. C., Jenkins, C. E., et al. (2018). Restoration of dendritic complexity, functional connectivity, and diversity of regenerated retinal bipolar neurons in adult zebrafish. J. Neurosci. 38, 120-136. doi: 10.1523/JNEUROSCI.3444-16. 2017

Mensinger, A. F., and Powers, M. K. (1999). Visual function in regenerating teleost retina following cytotoxic lesioning. Vis. Neurosci. 16, 241-251. doi: 10.1017/ S0952523899162059

Mensinger, A. F., and Powers, M. K. (2007). Visual function in regenerating teleost retina following surgical lesioning. Vis. Neurosci. 24, 299-307. doi: 10.1017/ S0952523807070265 
Miller, A. H., Howe, H. B., Krause, B. M., Friedle, S. A., Banks, M. I., Perkins, B. D., et al. (2018). Pregnancy associated plasma protein-aa (pappaa) regulates photoreceptor synaptic development to mediate visually guided behavior. J. Neurosci. 38, 5220-5236. doi: 10.1523/JNEUROSCI.0061-18.2018

Mills, J. O., Jalil, A., and Stanga, P. E. (2017). Electronic retinal implants and artificial vision: journey and present. Eye 31, 1383-1398. doi: 10.1038/eye.20 17.65

Mitchell, D. M., Lovel, A. G., and Stenkamp, D. L. (2018). Dynamic changes in microglial and macrophage characteristics during degeneration and regeneration of the zebrafish retina. J. Neuroinflammation 15:163. doi: 10.1186/ s12974-018-1185-6

Montgomery, J. E., Parsons, M. J., and Hyde, D. R. (2010). A novel model of retinal ablation demonstrates that the extent of rod cell death regulates the origin of the regenerated zebrafish rod photoreceptors. J. Comp. Neurol. 518, 800-814. doi: $10.1002 /$ cne. 22243

Moosajee, M., Gregory-Evans, K., Ellis, C. D., Seabra, M. C., and GregoryEvans, C. Y. (2008). Translational bypass of nonsense mutations in zebrafish rep1, pax2.1 and lamb1 highlights a viable therapeutic option for untreatable genetic eye disease. Hum. Mol. Genet. 17, 3987-4000. doi: 10.1093/hmg/ ddn 302

Morris, A. C., Scholz, T. L., Brockerhoff, S. E., and Fadool, J. M. (2008). Genetic dissection reveals two separate pathways for rod and cone regeneration in the teleost retina. Dev. Neurobiol. 68, 605-619. doi: 10.1002/dneu.20610

Moshiri, A., Gonzalez, E., Tagawa, K., Maeda, H., Wang, M., Frishman, L. J., et al. (2008). Near complete loss of retinal ganglion cells in the math5/brn3b double knockout elicits severe reductions of other cell types during retinal development. Dev. Biol. 316, 214-227. doi: 10.1016/j.ydbio.2008.01.015

Nagashima, M., Barthel, L. K., and Raymond, P. A. (2013). A self-renewing division of zebrafish Müller glial cells generates neuronal progenitors that require n-cadherin to regenerate retinal neurons. Development 140, 4510-4521. doi: 10.1242/dev.090738

Namdaran, P., Reinhart, K. E., Owens, K. N., Raible, D. W., and Rubel, E. W. (2012). Identification of modulators of hair cell regeneration in the zebrafish lateral line. J. Neurosci. 32, 3516-3528. doi: 10.1523/JNEUROSCI.3905-11.2012

Nelson, R., and Singla, N. (2009). A spectral model for signal elements isolated from zebrafish photopic electroretinogram. Vis. Neurosci. 26, 349-363. doi: $10.1017 /$ S0952523809990113

Nelson, S. M., Frey, R. A., Wardwell, S. L., and Stenkamp, D. L. (2008). The developmental sequence of gene expression within the rod photoreceptor lineage in embryonic zebrafish. Dev. Dyn. 237, 2903-2917. doi: 10.1002/dvdy. 21721

Neuhauss, S. C. (2003). Behavioral genetic approaches to visual system development and function in zebrafish. J. Neurobiol. 54, 148-160. doi: 10.1002/ neu. 10165

Neuhauss, S. C., Biehlmaier, O., Seeliger, M. W., Das, T., Kohler, K., Harris, W. A., et al. (1999). Genetic disorders of vision revealed by a behavioral screen of 400 essential loci in zebrafish. J. Neurosci. 19, 8603-8615. doi: 10.1523/JNEUROSCI. 19-19-08603.1999

Nicolson, T., Rüsch, A., Friedrich, R. W., Granato, M., Ruppersberg, J. P., NüssleinVolhard, C., et al. (1998). Genetic analysis of vertebrate sensory hair cell mechanosensation: the zebrafish circler mutants. Neuron 20, 271-283. doi: 10.1016/S0896-6273(00)80455-9

Noel, N. C. L., and Allison, W. T. (2018). Connectivity of cone photoreceptor telodendria in the zebrafish retina. J. Comp. Neurol. 526, 609-625. doi: 10.1002/ cne. 24354

Odermatt, B., Nikolaev, A., and Lagnado, L. (2012). Encoding of luminance and contrast by linear and nonlinear synapses in the retina. Neuron $73,758-773$. doi: 10.1016/j.neuron.2011.12.023

Orger, M. B., and Baier, H. (2005). Channeling of red and green cone inputs to the zebrafish optomotor response. Vis. Neurosci. 22, 275-281. doi: 10.1017/ S0952523805223039

Ortin-Martinez, A., Tsai, E. L., Nickerson, P. E., Bergeret, M., Lu, Y., Smiley, S., et al. (2017). A reinterpretation of cell transplantation: Gfp transfer from donor to host photoreceptors. Stem Cells 35, 932-939. doi: 10.1002/stem. 2552

Otteson, D. C., D'Costa, A. R., and Hitchcock, P. F. (2001). Putative stem cells and the lineage of rod photoreceptors in the mature retina of the goldfish. Dev. Biol. 232, 62-76. doi: 10.1006/dbio.2001.0163
Pardue, M. T., and Allen, R. S. (2018). Neuroprotective strategies for retinal disease. Prog. Retin. Eye Res. 65, 50-76. doi: 10.1016/j.preteyeres.2018.02.002

Patterson, B. W., Abraham, A. O., MacIver, M. A., and McLean, D. L. (2013). Visually guided gradation of prey capture movements in larval zebrafish. J. Exp. Biol. 216(Pt 16), 3071-3083. doi: 10.1242/jeb.087742

Pearson, R. A., Gonzalez-Cordero, A., West, E. L., Ribeiro, J. R., Aghaizu, N., Goh, D., et al. (2016). Donor and host photoreceptors engage in material transfer following transplantation of post-mitotic photoreceptor precursors. Nat. Commun. 7:13029. doi: 10.1038/ncomms13029

Pollak, J., Wilken, M. S., Ueki, Y., Cox, K. E., Sullivan, J. M., Taylor, R. J., et al. (2013). Ascll reprograms mouse Müller glia into neurogenic retinal progenitors. Development 140, 2619-2631. doi: 10.1242/dev.091355

Polosukhina, A., Litt, J., Tochitsky, I., Nemargut, J., Sychev, Y., De Kouchkovsky, I., et al. (2012). Photochemical restoration of visual responses in blind mice. Neuron 75, 271-282. doi: 10.1016/j.neuron.2012.05.022

Powell, C., Cornblath, E., Elsaeidi, F., Wan, J., and Goldman, D. (2016). Zebrafish Müller glia-derived progenitors are multipotent, exhibit proliferative biases and regenerate excess neurons. Sci. Rep. 6:24851. doi: 10.1038/srep24851

Ramachandran, R., Fausett, B. V., and Goldman, D. (2010a). Asclla regulates Müller glia dedifferentiation and retinal regeneration through a lin-28dependent, let-7 microRNA signalling pathway. Nat. Cell Biol. 12, 1101-1107. doi: $10.1038 /$ ncb 2115

Ramachandran, R., Reifler, A., Parent, J. M., and Goldman, D. (2010b). Conditional gene expression and lineage tracing of tubala expressing cells during zebrafish development and retina regeneration. J. Comp. Neurol. 518, 4196-4212. doi: $10.1002 /$ cne. 22448

Randlett, O., MacDonald, R. B., Yoshimatsu, T., Almeida, A. D., Suzuki, S. C., Wong, R. O., et al. (2013). Cellular requirements for building a retinal neuropil. Cell Rep. 3, 282-290. doi: 10.1016/j.celrep.2013.01.020

Rao, M. B., Didiano, D., and Patton, J. G. (2017). Neurotransmitter-regulated regeneration in the zebrafish retina. Stem Cell Rep. 8, 831-842. doi: 10.1016/j. stemcr.2017.02.007

Raymond, P. A., Barthel, L. K., Bernardos, R. L., and Perkowski, J. J. (2006). Molecular characterization of retinal stem cells and their niches in adult zebrafish. BMC Dev. Biol. 6:36. doi: 10.1186/1471-213X-6-36

Raymond, P. A., Barthel, L. K., and Stenkam, D. L. (1996). The zebrafish ultraviolet cone opsin reported previously is expressed in rods. Invest. Ophthalmol. Vis. Sci. 37, 948-950.

Raymond, P. A., Reifler, M. J., and Rivlin, P. K. (1988). Regeneration of goldfish retina: rod precursors are a likely source of regenerated cells. J. Neurobiol. 19, 431-463. doi: 10.1002/neu.480190504

Salbreux, G., Barthel, L. K., Raymond, P. A., and Lubensky, D. K. (2012). Coupling mechanical deformations and planar cell polarity to create regular patterns in the zebrafish retina. PLoS Comput. Biol. 8:e1002618. doi: 10.1371/journal.pcbi. 1002618

Santos-Ferreira, T., Llonch, S., Borsch, O., Postel, K., Haas, J., Ader, M., et al. (2016). Retinal transplantation of photoreceptors results in donor-host cytoplasmic exchange. Nat. Commun. 7:13028. doi: 10.1038/ncomms 13028

Santos-Ferreira, T., Postel, K., Stutzki, H., Kurth, T., Zeck, G., and Ader, M. (2015). Daylight vision repair by cell transplantation. Stem Cells 33, 79-90. doi: 10.1002/stem.1824

Sarria, I., Cao, Y., Stutzki, H., Kurth, T., Zeck, G., Ader, M., et al. (2018). Lrit1 modulates adaptive changes in synaptic communication of cone photoreceptors. Cell Rep. 22, 3562-3573. doi: 10.1016/j.celrep.2018.03.008

Schroeter, E. H., Wong, R. O. L., and Gregg, R. G. (2006). In vivo development of retinal on-bipolar cell axonal terminals visualized in nyx::Myfp transgenic zebrafish. Vis. Neurosci. 23, 833-843. doi: 10.1017/S095252380623 0219

Shah, A. N., Davey, C. F., Whitebirch, A. C., Miller, A. C., and Moens, C. B. (2016). Rapid reverse genetic screening using CRISPR in zebrafish. Zebrafish 13, 152-153. doi: 10.1089/zeb.2015.29000.sha

Shekhar, K., Lapan, S. W., Whitney, I. E., Tran, N. M., Macosko, E. Z., Kowalczyk, M., et al. (2016). Comprehensive classification of retinal bipolar neurons by single-cell transcriptomics. Cell 166, 1308-1323.e30. doi: 10.1016/ j.cell.2016.07.054

Sherpa, T., Fimbel, S. M., Mallory, D. E., Maaswinkel, H., Spritzer, S. D., Sand, J. A., et al. (2008). Ganglion cell regeneration following whole-retina destruction in zebrafish. Dev. Neurobiol. 68, 166-181. doi: 10.1002/dneu.20568 
Sherpa, T., Lankford, T., McGinn, T. E., Hunter, S. S., Frey, R. A., Sun, C., et al. (2014). Retinal regeneration is facilitated by the presence of surviving neurons. Dev. Neurobiol. 74, 851-876. doi: 10.1002/dneu.22167

Shi, Y., Obert, E., Rahman, B., Rohrer, B., and Lobo, G. P. (2017). The retinol binding protein receptor $2(\mathrm{rbpr} 2)$ is required for photoreceptor outer segment morphogenesis and visual function in zebrafish. Sci. Rep. 7:16207. doi: 10.1038/ s41598-017-16498-9

Silver, P. H. (1974). Photopic spectral sensitivity of the neon tetra [Paracheirodon innesi (Myers)] found by the use of a dorsal light reaction. Vis. Res. 14, 329-334. doi: 10.1016/0042-6989(74)90091-1

Simmons, A. B., Bloomsburg, S. J., Sukeena, J. M., Miller, C. J., Ortega-Burgos, Y., Borghuis, B. G., et al. (2017). DSCAM-mediated control of dendritic and axonal arbor outgrowth enforces tiling and inhibits synaptic plasticity. Proc. Natl. Acad. Sci. U.S.A. 114, E10224-E10233. doi: 10.1073/pnas.1713548114

Singh, M. S., Balmer, J., Barnard, A. R., Aslam, S. A., Moralli, D., Green, C. M., et al. (2016). Transplanted photoreceptor precursors transfer proteins to host photoreceptors by a mechanism of cytoplasmic fusion. Nat. Commun. 7:13537. doi: $10.1038 /$ ncomms 13537

Singh, M. S., Charbel Issa, P., Butler, R., Martin, C., Lipinski, D. M., Sekaran, S., et al. (2013). Reversal of end-stage retinal degeneration and restoration of visual function by photoreceptor transplantation. Proc. Natl. Acad. Sci. U.S.A. 110, 1101-1106. doi: 10.1073/pnas.1119416110

Smiley, S., Nickerson, P. E., Comanita, L., Daftarian, N., El-Sehemy, A., Tsai, E. L., et al. (2016). Establishment of a cone photoreceptor transplantation platform based on a novel cone-gfp reporter mouse line. Sci. Rep. 6:22867. doi: 10.1038/ srep 22867

Smyth, V. A., Di Lorenzo, D., and Kennedy, B. N. (2008). A novel, evolutionarily conserved enhancer of cone photoreceptor-specific expression. J. Biol. Chem. 283, 10881-10891. doi: 10.1074/jbc.M710454200

Solin, S. L., Wang, Y., Mauldin, J., Schultz, L. E., Lincow, D. E., Brodskiy, P. A., et al. (2014). Molecular and cellular characterization of a zebrafish optic pathway tumor line implicates glia-derived progenitors in tumorigenesis. PLoS One 9:e114888. doi: 10.1371/journal.pone.0114888

Stearns, G., Evangelista, M., Fadool, J. M., and Brockerhoff, S. E. (2007). A mutation in the cone-specific pde6 gene causes rapid cone photoreceptor degeneration in zebrafish. J. Neurosci. 27, 13866-13874. doi: 10.1523/JNEUROSCI.3136-07. 2007

Stenkamp, D. L. (2015). Development of the vertebrate eye and retina. Prog. Mol. Biol. Transl. Sci. 134, 397-414. doi: 10.1016/bs.pmbts.2015.06.006

Stenkamp, D. L., and Cameron, D. A. (2002). Cellular pattern formation in the retina: retinal regeneration as a model system. Mol. Vis. 8, 280-293.

Stenkamp, D. L., Powers, M. K., Carney, L. H., and Cameron, D. A. (2001). Evidence for two distinct mechanisms of neurogenesis and cellular pattern formation in regenerated goldfish retinas. J. Comp. Neurol. 431, 363-381. doi: 10.1002/1096-9861(20010319)431:4<363::AID-CNE1076>3.0.CO;2-7

Stuermer, C. A. O., Niepenberg, A., and Wolburg, H. (1985). Aberrant axonal paths in regenerated goldfish retina and tectum opticum following intraocular injection of ouabain. Neurosci. Lett. 58, 333-338. doi: 10.1016/0304-3940(85) 90076-X

Sun, C., Galicia, C., and Stenkamp, D. L. (2018). Transcripts within rod photoreceptors of the zebrafish retina. BMC Genomics 19:127. doi: 10.1186/ s12864-018-4499-y

Suzuki, S. C., Bleckert, A., Williams, P. R., Takechi, M., Kawamura, S., Wong, R. O., et al. (2013). Cone photoreceptor types in zebrafish are generated by symmetric terminal divisions of dedicated precursors. Proc. Natl. Acad. Sci. U.S.A. 110, 15109-15114. doi: 10.1073/pnas.1303551110

Takechi, M., Hamaoka, T., and Kawamura, S. (2003). Fluorescence visualization of ultraviolet-sensitive cone photoreceptor development in living zebrafish. FEBS Lett. 553, 90-94. doi: 10.1016/S0014-5793(03)00977-3

Takechi, M., Seno, S., and Kawamura, S. (2008). Identification of cis-acting elements repressing blue opsin expression in zebrafish uv cones and pineal cells. J. Biol. Chem. 283, 31625-31632. doi: 10.1074/jbc.M806226200

Tappeiner, C., Balmer, J., Iglicki, M., Schuerch, K., Jazwinska, A., Enzmann, V., et al. (2013). Characteristics of rod regeneration in a novel zebrafish retinal degeneration model using n-methyl-n-nitrosourea (mnu). PLoS One 8:e71064. doi: 10.1371/journal.pone.0071064

Thomas, J. L., Nelson, C. M., Luo, X., Hyde, D. R., and Thummel, R. (2012). Characterization of multiple light damage paradigms reveals regional differences in photoreceptor loss. Exp. Eye Res. 97, 105-116. doi: 10.1016/j.exer. 2012.02.004

Thomas, J. L., Ranski, A. H., Morgan, G. W., and Thummel, R. (2016). Reactive gliosis in the adult zebrafish retina. Exp. Eye Res. 143, 98-109. doi: 10.1016/j. exer.2015.09.017

Tochitsky, I., Trautman, J., Gallerani, N., Malis, J. G., and Kramer, R. H. (2017). Restoring visual function to the blind retina with a potent, safe and long-lasting photoswitch. Sci. Rep. 7:45487. doi: 10.1038/srep45487

Torvund, M. M., Ma, T. S., Connaughton, V. P., Ono, F., and Nelson, R. F. (2017). Cone signals in monostratified and bistratified amacrine cells of adult zebrafish retina. J. Comp. Neurol. 525, 2800-2801. doi: 10.1002/cne.24227

Trifunovic, D., Sahaboglu, A., Kaur, J., Mencl, S., Zrenner, E., Ueffing, M., et al. (2012). Neuroprotective strategies for the treatment of inherited photoreceptor degeneration. Curr. Mol. Med. 12, 598-612. doi: 10.2174/156652412800620048

Tsujimura, T., Chinen, A., and Kawamura, S. (2007). Identification of a locus control region for quadruplicated green-sensitive opsin genes in zebrafish. Proc. Natl. Acad. Sci. U.S.A. 104, 12813-12818. doi: 10.1073/pnas.0704061104

Tsujimura, T., Hosoya, T., and Kawamura, S. (2010). A single enhancer regulating the differential expression of duplicated red-sensitive opsin genes in zebrafish. PLoS Genet. 6:e1001245. doi: 10.1371/journal.pgen.1001245

Ueki, Y., Wilken, M. S., Cox, K. E., Chipman, L., Jorstad, N., Sternhagen, K., et al. (2015). Transgenic expression of the proneural transcription factor ascl1 in Müller glia stimulates retinal regeneration in young mice. Proc. Natl. Acad. Sci. U.S.A. 112, 13717-13722. doi: 10.1073/pnas.1510595112

Ueno, A., Omori, Y., Sugita, Y., Watanabe, S., Chaya, T., Kozuka, T., et al. (2018). Lrit1, a retinal transmembrane protein, regulates selective synapse formation in cone photoreceptor cells and visual acuity. Cell Rep. 22, 3548-3561. doi: 10.1016/j.celrep.2018.03.007

Varshney, G. K., Carrington, B., Pei, W., Bishop, K., Chen, Z., Fan, C., et al. (2016). A high-throughput functional genomics workflow based on crispr/cas9mediated targeted mutagenesis in zebrafish. Nat. Protoc. 11, 2357-2375. doi: 10.1038/nprot.2016.141

Varshney, G. K., Pei, W., LaFave, M. C., Idol, J., Xu, L., Gallardo, V., et al. (2015). High-throughput gene targeting and phenotyping in zebrafish using crispr/cas9. Genome Res. 25, 1030-1042. doi: 10.1101/gr.186379.114

Veldman, M. B., Bemben, M. A., Thompson, R. C., and Goldman, D. (2007). Gene expression analysis of zebrafish retinal ganglion cells during optic nerve regeneration identifies klf6a and klf7a as important regulators of axon regeneration. Dev. Biol. 312, 596-612. doi: 10.1016/j.ydbio.2007.09.019

Vihtelic, T. S., Doro, C. J., and Hyde, D. R. (1999). Cloning and characterization of six zebrafish photoreceptor opsin cDNAS and immunolocalization of their corresponding proteins. Vis. Neurosci. 16, 571-585. doi: 10.1017/ S0952523899163168

Vihtelic Thomas, S., and Hyde David, R. (2000). Light-induced rod and cone cell death and regeneration in the adult albino zebrafish (danio rerio) retina. J. Neurobiol. 44, 289-307. doi: 10.1002/1097-4695(20000905)44:3<289::AID$\mathrm{NEU} 1>3.0 . \mathrm{CO} ; 2-\mathrm{H}$

Vitorino, M., Jusuf, P. R., Maurus, D., Kimura, Y., Higashijima, S.-I., and Harri, W. A. (2009). Vsx2 in the zebrafish retina: restricted lineages through derepression. Neural Dev. 4:14. doi: 10.1186/1749-8104-4-14

Wahlin, K. J., Maruotti, J. A., Maurus, D., Kimura, Y., Higashijima, S., and Harris, W. A. (2017). Photoreceptor outer segment-like structures in long-term 3d retinas from human pluripotent stem cells. Sci. Rep. 7:766. doi: 10.1038/s41598017-00774-9

Waldron, P. V., Di Marco, F., Kruczek, K., Ribeiro, J., Graca, A. B., Hippert, C., et al. (2018). Transplanted donor- or stem cell-derived cone photoreceptors can both integrate and undergo material transfer in an environment-dependent manner. Stem Cell Rep. 10, 406-421. doi: 10.1016/j.stemcr.2017.12.008

Wan, J., and Goldman, D. (2016). Retina regeneration in zebrafish. Curr. Opin. Genet. Dev. 40, 41-47. doi: 10.1016/j.gde.2016.05.009

Wan, J., Zhao, X.-F., Vojtek, A., and Goldman, D. (2014). Retinal injury, growth factors and cytokines converge on $\beta$-catenin and pstat 3 signaling to stimulate retina regeneration. Cell Rep. 9, 285-297. doi: 10.1016/j.celrep.2014. 08.048

Wan, J., Zheng, H., Chen, Z. L., Xiao, H. L., Shen, Z. J., Zhou, G. M., et al. (2008). Preferential regeneration of photoreceptor from Müller glia after retinal degeneration in adult rat. Vis. Res. 48, 223-234. doi: 10.1016/j.visres.2007. 11.002 
Wang, Y., Fehlhaber, K. E., Sarria, I., Cao, Y., Ingram, N. T., Guerrero-Given, D., et al. (2017). The auxiliary calcium channel subunit alpha2delta4 is required for axonal elaboration, synaptic transmission, and wiring of rod photoreceptors. Neuron 93, 1359-1374.e6. doi: 10.1016/j.neuron.2017.02.021

Warwick, A., and Lotery, A. (2018). Genetics and genetic testing for age-related macular degeneration. Eye 32, 849-857. doi: 10.1038/eye.2017.245

Weber, I. P., Ramos, A. P., Strzyz, P. J., Leung, L. C., Young, S., and Norden, C. (2014). Mitotic position and morphology of committed precursor cells in the zebrafish retina adapt to architectural changes upon tissue maturation. Cell Rep. 7, 386-397. doi: 10.1016/j.celrep.2014.03.014

White, D. T., Sengupta, S., Saxena, M. T., Xu, Q., Hanes, J., Ding, D., et al. (2017). Immunomodulation-accelerated neuronal regeneration following selective rod photoreceptor cell ablation in the zebrafish retina. Proc. Natl. Acad. Sci. U.S.A. 114, E3719-E3728. doi: 10.1073/pnas.1617721114

White, R. M., Sessa, A., Burke, C., Bowman, T., LeBlanc, J., Ceol, C., et al. (2008). Transparent adult zebrafish as a tool for in vivo transplantation analysis. Cell Stem Cell 2, 183-189. doi: 10.1016/j.stem.2007.11.002

Williams, P. R., Suzuki, S. C., Yoshimatsu, T., Lawrence, O. T., Waldron, S. J., Parsons, M. J., et al. (2010). In vivo development of outer retinal synapses in the absence of glial contact. J. Neurosci. 30, 11951-11961. doi: 10.1523/ JNEUROSCI.3391-10.2010

Yazulla, S., and Studholme, K. M. (2001). Neurochemical anatomy of the zebrafish retina as determined by immunocytochemistry. J. Neurocytol. 30, 551-592. doi: 10.1023/A:1016512617484

Yin, J., Brocher, J., Linder, B., Hirmer, A., Sundaramurthi, H., Fischer, U., et al. (2012). The 1d4 antibody labels outer segments of long double cone but not rod photoreceptors in zebrafish. Invest. Ophthalmol. Vis. Sci. 53, 4943-4951. doi: 10.1167/iovs.12-9511

Yoshimatsu, T., D’Orazi, F. D., Gamlin, C. R., Suzuki, S. C., Suli, A., Kimelman, D., et al. (2016). Presynaptic partner selection during retinal circuit reassembly varies with timing of neuronal regeneration in vivo. Nat. Commun. 7:10590. doi: $10.1038 /$ ncomms 10590
Yue, L., Weiland, J. D., Roska, B., and Humayun, M. S. (2016). Retinal stimulation strategies to restore vision: fundamentals and systems. Prog. Retin. Eye Res. 53, 21-47. doi: 10.1016/j.preteyeres.2016.05.002

Zhang, C., Kolodkin, A. L., Wong, R. O., and James, R. E. (2017). Establishing wiring specificity in visual system circuits: from the retina to the brain. Annu. Rev. Neurosci. 40, 395-424. doi: 10.1146/annurev-neuro-072116-03 1607

Zhang, Q. X., He, X. J., Wong, H. C., and Kindt, K. S. (2016). Functional calcium imaging in zebrafish lateral-line hair cells. Methods Cell. Biol. 133, 229-252. doi: 10.1016/bs.mcb.2015.12.002

Zhang, Y., Yang, Y., Trujillo, C., Zhong, W., and Leung, Y. F. (2012). The expression of irx7 in the inner nuclear layer of zebrafish retina is essential for a proper retinal development and lamination. PLoS One 7:e36145. doi: 10.1371/journal. pone.0036145

Zhao, X. F., Ellingsen, S., and Fjose, A. (2009). Labelling and targeted ablation of specific bipolar cell types in the zebrafish retina. BMC Neurosci. 10:107. doi: 10.1186/1471-2202-10-107

Zhong, X., Gutierrez, C., Xue, T., Hampton, C., Vergara, M. N., Cao, L. H., et al. (2014). Generation of three dimensional retinal tissue with functional photoreceptors from human ipscs. Nat. Commun. 5:4047. doi: 10.1038/ ncomms5047

Conflict of Interest Statement: The authors declare that the research was conducted in the absence of any commercial or financial relationships that could be construed as a potential conflict of interest.

Copyright (c) 2018 Angueyra and Kindt. This is an open-access article distributed under the terms of the Creative Commons Attribution License (CC BY). The use, distribution or reproduction in other forums is permitted, provided the original author(s) and the copyright owner(s) are credited and that the original publication in this journal is cited, in accordance with accepted academic practice. No use, distribution or reproduction is permitted which does not comply with these terms. 